\title{
Meta-Analysis of the Relationship Between the Big Five and Academic Success at University
}

\author{
Sabrina Trapmann, Benedikt Hell, Jan-Oliver W. Hirn, and Heinz Schuler
}

Department of Psychology, University of Hohenheim, Stuttgart, Germany

\begin{abstract}
Interest in the prediction of academic success in higher education has grown considerably in recent years in German-speaking countries. While the validity of school grades and admission tests has been investigated by meta-analyses and large-scale studies at least in the United States, less is known about noncognitive predictors of academic success. The present meta-analysis investigates the impact of the Big Five personality factors on academic success at university. A total of 258 correlation coefficients from 58 studies published since 1980 were included. Grades, retention, and satisfaction served as success criteria. Correlations were corrected for attenuation caused by measurement error. Results show that the influence of personality traits on academic achievement depends on the success criterion. While Neuroticism is related to academic satisfaction $\left(\hat{\mu}_{\rho}=-.369, k=8\right)$, Conscientiousness correlates with grades $\left(\hat{\mu}_{\rho}=.269, k=41\right)$. Extraversion, Openness to Experience, and Agreeableness have no significant impact on academic success. Moderator analyses suggest effects of culture for the validity of Extraversion. Parallels to validity for job performance are identified and implications for admission and counseling of students are discussed.
\end{abstract}

Keywords: meta-analysis, academic performance prediction, personality, Big Five personality factors, academic admission criteria

\section{Introduction}

Several meta-analyses have investigated the predictive validity of different college admission tests, achievement tests, and high school grades. The validity of college admission tests such as the SAT (Scholastic Aptitude Test) or GRE (Graduate Record Examination), which are frequently used in the United States, has been examined by large scale studies and meta-analyses. Burton and Ramist (2001) report a mean correlation coefficient of .36 between SAT-scores and college GPA. Kuncel, Hezlett, and Ones (2001) calculated mean correlation coefficients between the different parts of GRE and graduate GPA up to .49. Hell, Trapmann, and Schuler (2007) meta-analyzed the validity of subject-specific academic aptitude tests in German-speaking countries and found a mean correlation coefficient of .48. Predictive validity of general mental ability seems to be slightly lower. Kuncel, Hezlett, and Ones (2004) analyzed the relationship between the Miller Analogies Test (MAT) and graduate GPA and found a mean correlation coefficient of .39. For school grades, meta-analyses estimate the mean correlation to college grades to be about 40 to .50 . Trapmann, Hell, Weigand, and Schuler (2007) report a corrected predictive validity of .53 for German school grades. Schuler, Funke, and Baron-Boldt (1990) calculated mean corrected correlations up to .56 for economic study majors for the same relationship. The meta-analysis calculated by Burton and
Ramist (2001) estimates the predictive validity of school grades for college grades in the United States at .42. All these figures indicate strong relationships regarding multiple influences of time and conditions.

In sum, the predictive validity evidence supporting relations between achievement measures and school grades is well confirmed. Nevertheless, only about $25 \%$ of the variance in college GPA can be explained (e.g., Tross, Harper, Osher, \& Kneidinger, 2000). Hence, there might be additional variables influencing academic performance. While many studies investigate the correlations between Big Five personality factors and academic achievement, there are very few meta-analyses on noncognitive measures. Most of them explore only a single factor or one single facet of a factor as, for example, anxiety (Seipp, 1991) or achievement motivation (Robbins, Lauver, Le, Davis, Langley, \& Carlstrom, 2004). Furthermore, many studies consider a single criterion of academic success, namely grades, retention, or satisfaction. In most of the cases grades are used to operationalize academic success. In fact, there are very view empirical studies considering any other criterion of college success, although this has been described more broadly in college handbooks, mission statements, and academic discussions (Camara, 2005). Correlations between grades and other measures of academic success such as satisfaction or retention are seldom reported in the literature. Trapmann (2007) found rather small correlations between grades and several other criteria of academic success such as satisfac- 
tion, task performance, or organizational citizenship performance.

Some studies have shown that faculty members believe that noncognitive factors, such as motivation, conscientiousness, perseverance, or helpfulness are important parts of academic success (Taber \& Hackman, 1976; Reilly, 1976; Willingham, 1985; Enright \& Gitomer, 1989; Oswald, Schmitt, Kim, Ramsay, \& Gillespie, 2004). Kyllonen, Walters, and Kaufman (2005) hypothesize that using noncognitive factors in addition to cognitive factors could increase fairness in admission decisions for graduate education, because gender and race differences tend to be smaller in noncognitive factors than in cognitive factors (for an opposing view see Potosky, Bobko, \& Roth, 2005). Goldberg (2001) noted that including measures of personality in university admission procedures could increase diversity, which is diminished by using primarily cognitive tasks. However, the use of personality measures in admission procedures might lead to new problems. One of these difficulties is faking. Personality traits are typically measured by self-reports, which enable applicants to give false information, and - most importantly - the impact of noncognitive traits is still unclear.

The present study reports a meta-analytic calculation of the correlation coefficients between the Big Five personality factors (five-factor model of personality) and academic success with the aspects: academic achievement (defined by university grades), retention, and satisfaction. Different conceivable moderator variables such as publication date, country of origin, study major, source of publication, age, study period (Bachelor vs. Master, basic vs. main studies), and heterogeneity of the sample were also considered. The results should show whether individual differences concerning these personality traits influence students' academic performance, satisfaction, and retention.

\section{The Big Five Framework}

Although there is some disagreement regarding designations and definitions of the Big Five personality factors (Digman, 1990), they can generally be defined as follows (Costa \& McCrae, 1992a). Neuroticism is a measure of emotional stability vs. instability. Emotional Stability might be manifested in students' responding appropriately to stress and tight time deadlines and in their adaptability to new situations or conditions (Goldberg, 2001). Anxiety, angry hostility, depression, self-consciousness, impulsivity, and vulnerability are the facets of this dimension as described by Costa and McCrae (1992b). Extraversion (or "surgency") is defined as the quantity and intensity of interpersonal interaction, encompassing traits such as assertiveness, sociability, activity, cheerfulness, and gregariousness. Hogan (1986) suggested that this dimension can be interpreted as ambition (initiative, surgency, ambition, and impetuousness), on the one hand, and sociability (sociable, exhibitionist, and expressive) on the other. The six lower-level traits in the model of Costa and McCrae (1992b) are: warmth, gregariousness, assertiveness, activity, excitement-seeking, and positive emotions. Imagination, creativity, curiosity, originality, and artistic sensibility are associated with Openness to Experience (also called intellect or culture), which consists of the facets openness to fantasy, to esthetics, to feelings, to actions, to ideas, and to values (Costa \& McCrae, 1992b). Agreeableness (or likability) is associated with being courteous, flexible, trusting, cooperative, tolerant, and treating others fairly and kindly. Costa and McCrae (1992b) mention the traits trust, straightforwardness, altruism, compliance, modesty, and tender-mindedness. Finally, the individual degrees of dependability, organization, persistence, and achievement-orientation determine a person's Conscientiousness. The six facets in the model of Costa and McCrae (1992b) are competence, order, dutifulness, achievement striving, self-discipline, and deliberation. Taken together, the five-factor model provides a comprehensive and parsimonious theoretical framework (Costa \& McCrae, 1992a) allowing for systematic reviews and meta-analyses.

\section{The Big Five and Job Performance}

Notwithstanding the lack of meta-analyses on the relationship of the Big Five and academic success, there are several meta-analyses that focus on the prediction of job performance (e.g., Barrick \& Mount, 1991; Tett, Jackson, \& Rothstein, 1991; Salgado, 1997). Barrick, Mount, and Judge (2001) quantitatively summarize the results of 15 meta-analytic studies that investigated the relationship between the Big Five and job performance. This secondorder meta-analysis revealed that Conscientiousness is a valid predictor of performance across several performance measures in a wide range of occupations. Emotional Stability (Neuroticism) was also found to be a generalizable predictor when overall work performance was the criterion. The remaining three traits of the five-factor model (Extraversion, Openness to Experience, and Agreeableness) did not predict overall work performance, but they did predict success in specific occupations or were related to specific criteria. The criterion with the highest correspondence to academic performance at university in this study was training performance. Barrick et al. (2001) found a different validitypattern for this criterion: Openness to Experience $\left(\hat{\mu}_{\rho}=\right.$ $.24)$, Conscientiousness $\left(\hat{\mu}_{\rho}=.23\right)$, and Extraversion $\left(\hat{\mu}_{\rho}=.23\right)$ demonstrated substantial validity. Emotional Stability $\left(\hat{\mu}_{\rho}=.08\right)$ and Agreeableness $\left(\hat{\mu}_{\rho}=.11\right)$ were less valid predictors. One topic of the present meta-analysis is to investigate whether the results for training performance in the job context are true for success in learning at college and university as well. 


\section{The Big Five and Academic Achievement}

Existing results concerning the relationship between the Big Five and academic achievement provide a wide range of coefficients. Some studies report correlations up to .40 for Conscientiousness (Furnham, Chamorro-Premuzic, \& McDougall, 2002) and up to .42 for Openness to Experience (Gakhar, 1986) or even up to .78 for Extraversion (Huq, Rabman, \& Mahmud, 1986; see Table 1). These results suggest a remarkable impact of personality traits on academic achievement. Some authors report incremental validities of personality over and above academic ability tests (such as the SAT) and recommend using personality assessment for college admissions (e.g., Conscientiousness: Conard, 2006). Following these results, personality measures are promising predictors of academic outcome and should, therefore, be applied to university student admission. On the other hand, some studies report opposite relations and negative correlation coefficients down to -.32 for Extraversion (Giesen, Gold, Hummer, \& Jansen, 1986) or -.21 for Openness to Experience (Gakhar, 1986).

De Raad and Schouwenburg (1996) reviewed the role of personality traits in learning and education and assumed that Extraversion is negatively related to success at the university level. They drew a rather ambiguous picture regarding Agreeableness and concluded that constructive internalization of social norms is a positive factor in academic achievement (p. 325). De Raad and Schouwenburg state that Conscientiousness and Openness to Experience represent the traits that are a main psychological resource in learning and education and are valid in predicting college performance. High Neuroticism, on the other hand, leads to poorer college performance. There is empirical evidence for a negative correlation of the facet anxiety and academic achievement. Seipp (1991) found a validity of $\hat{\mu}_{\rho}=-.212$ concerning anxiety and academic performance of children, adolescents, and adults. Goldberg (2001) found Conscientiousness to be a useful predictor of academic performance. He stresses the impact of certain aspects of Conscientiousness, including the need for achievement, organization, efficiency, carefulness, and dependability. In his view, Emotional Stability might be important, too, while Extraversion and Agreeableness have little influence on academic success (Goldberg, 2001).

\section{Hypotheses}

Given the results on training performance and the findings of De Raad and Schouwenburg (1996), we expect substantial validity of Conscientiousness and Openness to Experience for college grades. These hypotheses correspond with the suggestions of Goldberg (2001). We expect small positive validities for Emotional Stability and
Agreeableness. The relation of achievement and Extraversion appears to be ambiguous. In light of the comparatively stronger empirical evidence from the studies on training performance (Barrick et al., 2001) we expect a positive validity of Extraversion for college grades. Goldberg (2001) suggested that Extraversion might predict success in some kinds of academic contexts.

Hypotheses for the criterion domain retention are more difficult to develop. The findings of research on job turnover/tenure may provide some first indications: In their meta-analysis, Barrick and Mount (1991) found small validity coefficients for the Big Five and job turnover/tenure. Openness to Experience was correlated negatively $\left(\hat{\mu}_{\rho}=-.11\right)$ with tenure and Conscientiousness positively $\left(\mu_{\rho}=.12\right)$. These results may be cues for an expected validity pattern in an academic context, although dropping out of college and quitting a job may be similar but not identical life events. However, Conscientiousness (covering facets like endurance and achievement motivation) may, plausibly, reduce college dropout. On the other hand, students with high Openness to Experience, such as curiosity, will rather tend to change their study major. Hence, we hypothesize that Conscientiousness (positively) and Openness to Experience (negatively) show small but significant validities for academic retention.

Regarding academic satisfaction, it is conceivable to draw comparisons with life satisfaction and job satisfaction. Neuroticism is the strongest predictor among the Big Five for life satisfaction as well as for job satisfaction (DeNeve \& Cooper, 1998; Judge, Heller, \& Mount, 2002). Correlation coefficients between Openness to Experience and job satisfaction and Openness to Experience and life satisfaction, respectively, are reported to be very low. (DeNeve \& Cooper, 1998). Results for the criteria life satisfaction and job satisfaction are very similar despite all differences in content. Hence, similar results for academic satisfaction can be assumed as well. We, therefore, expect positive validities especially for Emotional Stability and smaller positive correlations with the four other traits.

In summary, the following hypotheses will be tested in this study: Conscientiousness and Openness to Experience are expected to be valid predictors for academic success; operationalized by grades, retention, and satisfaction. Conscientiousness contains those traits that are important for accomplishing tasks in general, such as being orderly, disciplined, ambitious, or persevering. Openness to Experience relates to characteristics that facilitate learning but may hinder maintenance until graduation. Extraversion and Agreeableness are expected to be correlated with college grades and satisfaction as, for example, gregarious and tender-minded students will socialize more easily with other students as well as with teachers and professors. We expected Neuroticism to be negatively correlated with satisfaction and, to a smaller extent, with grades. 
Predictors

Criteria

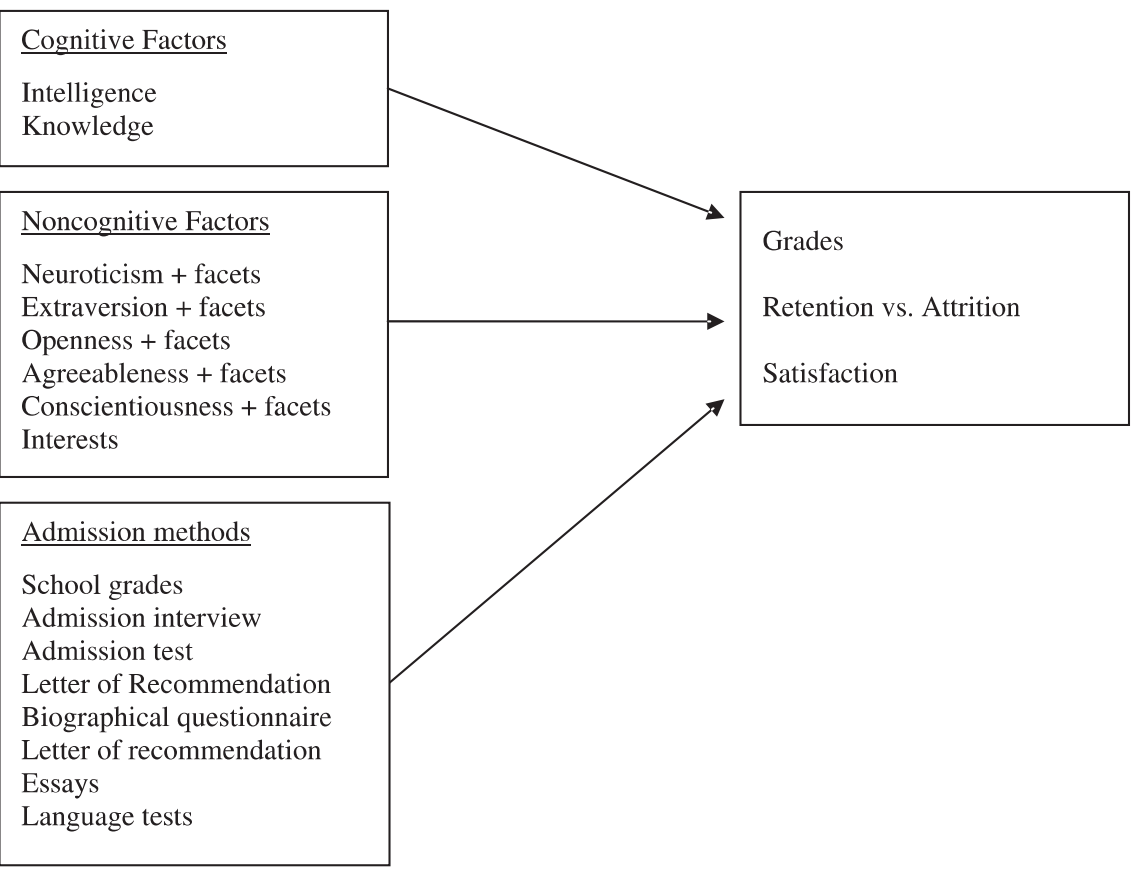

Figure 1. Meta-analytic investigated predictors and criteria of academic success in higher education.

\section{Method}

This meta-analysis was part of a larger meta-analytic project. We investigated bivariate relations between several predictors and different criteria of academic success in higher education. We sought to find all studies published since 1980 that reported empirical results for the relationships between the following predictors and criteria (Figure 1): school grades, admission interviews, biographical questionnaires, letters of recommendation, essays, intelligence, admission tests, knowledge tests, language tests, personality traits, and interests; success criteria were: grades, retention vs. attrition, and satisfaction. European studies were included concerning the predictors school grades, intelligence, admission, and knowledge tests, because there are already meta-analyses for North American results (e.g., Burton \& Ramist, 2001; Kuncel et al., 2001, 2004). Concerning all other predictors, studies from all over the world were included whether they were published in English or in German.

The present meta-analysis examined the validity of personality traits for academic success at university. We chose the five-factor model as an integrating framework to describe and aggregate the empirical results. We found a few studies presenting the validity of traits too narrow to subsume them under one of the Big Five. These traits were coded and meta-analytically aggregated if they fit the Big Five facets of Costa and McCrae (1992b) sufficiently. Costa and McCrae (1992b) have presented a taxonomy that subsumes six lower-level traits (referred to as facets) under each of the Big Five personality factors, such as anxiety for Neuroticism or altruism for Agreeableness. The results for the lower-level traits will be presented in this paper when they provide useful additional information.

\section{Literature Search}

Several sources were searched to identify all studies since 1980 that examined the relationship between personality traits and grades, retention, and satisfaction. Electronic searches were performed on PsycInfo (1980-2004) and Psyndex (1980-2004). The citation lists of all obtained articles and reports were then inspected manually to identify further promising studies. Additionally, colleagues working in relevant fields were contacted. The following types of studies were excluded from further review: (1) studies reporting no empirical results; (2) studies failing to report zero-order correlations or statistics that can be converted into correlations (e.g., studies only reporting partial correlations, multiple regression $\beta$, significance level); (3) studies reporting only results of extreme groups (e.g., examining academic success of highly anxious students only or comparing of the best with the worst students); (4) studies investigating samples not from tertiary education (e.g., high school students; apprentices); (5) studies examining predictors that do not definitely fit one of the Big Five personality traits; (6) studies available in neither English nor German; (7) studies using other criteria than grades, retention information, or self-reports of satisfaction. 
Table 1. Number of validity coefficients and sample sizes for each predictor-criterion combination

\begin{tabular}{|c|c|c|c|c|c|c|}
\hline Predictor & $N$ & $k$ & $M$ & $S D$ & Min & Max \\
\hline \multicolumn{7}{|c|}{ Criterion academic achievement (grades): } \\
\hline Neuroticism & 14653 & 59 & -.04 & .14 & -.37 & .22 \\
\hline Extraversion & 12424 & 54 & .01 & .21 & -.32 & .78 \\
\hline Openness to Experience & 14942 & 41 & .08 & .14 & -.21 & .42 \\
\hline Agreeableness & 12452 & 34 & .04 & .10 & -.23 & .25 \\
\hline Conscientiousness & 10855 & 41 & .22 & .12 & -.10 & .40 \\
\hline \multicolumn{7}{|l|}{ Criterion retention: } \\
\hline Neuroticism & 2714 & 4 & -.02 & .08 & -.10 & .08 \\
\hline Extraversion & 3916 & 5 & .00 & .04 & -.06 & .04 \\
\hline Openness to Experience & 2194 & 4 & -.02 & .09 & -.13 & .05 \\
\hline \multicolumn{7}{|l|}{ Criterion satisfaction: } \\
\hline Neuroticism & 1512 & 8 & -.27 & .04 & -.31 & -.17 \\
\hline Extraversion & 660 & 4 & .06 & .21 & -.26 & .17 \\
\hline
\end{tabular}

Notes: $N=$ overall sample size, $k=$ number of independent samples $=$ number of analyzed validity coefficients, $M=$ mean observed correlation, $S D=$ standard deviation of the correlation, Min = minimum of the correlation, Max = maximum of the correlation.

Table 2. Summary of study characteristics

\begin{tabular}{|c|c|c|c|c|c|c|c|c|c|}
\hline Author(s) & Country & Predictor & Criterion & $N$ & $A$ & $F$ & Study major & Study level & $r$ \\
\hline Barchard (2003) & USA & $\begin{array}{l}\text { Neuroticism } \\
\text { Extraversion } \\
\text { Openness } \\
\text { Agreeableness } \\
\text { Conscientiousness }\end{array}$ & GPA & 150 & 21.5 & 62.7 & Multiple majors & Bachelor & $\begin{array}{r}-.12 \\
.05 \\
.17 \\
.14 \\
.33\end{array}$ \\
\hline $\begin{array}{l}\text { Bauer \& Liang } \\
(2003)\end{array}$ & USA & $\begin{array}{l}\text { Neuroticism } \\
\text { Extraversion } \\
\text { Openness } \\
\text { Agreeableness } \\
\text { Conscientiousness }\end{array}$ & GPA & 265 & - & 58.5 & Multiple majors & Bachelor & $\begin{array}{r}.00 \\
-.18 \\
-.02 \\
.06 \\
.22\end{array}$ \\
\hline $\begin{array}{l}\text { Brandstätter \& } \\
\text { Farthofer (2002) }\end{array}$ & Austria & $\begin{array}{l}\text { Extraversion } \\
\text { Conscientiousness }\end{array}$ & GPA & 705 & 19.0 & - & Multiple majors & Main study period & $\begin{array}{r}-.26 \\
.08\end{array}$ \\
\hline $\begin{array}{l}\text { Busato, Prins, } \\
\text { Elshout, \& } \\
\text { Hamaker (2000) }\end{array}$ & Netherlands & $\begin{array}{l}\text { Neuroticism } \\
\text { Extraversion } \\
\text { Openness } \\
\text { Agreeableness } \\
\text { Conscientiousness }\end{array}$ & GPA & 409 & - & - & Psychology & Basic study period & $\begin{array}{r}.06 \\
-.13 \\
.03 \\
.00 \\
.16\end{array}$ \\
\hline \multirow[t]{2}{*}{$\begin{array}{l}\text { Chamorro-Pre- } \\
\text { muzic \& Furnham } \\
\text { (2003a) }\end{array}$} & UK & $\begin{array}{l}\text { Neuroticism } \\
\text { Extraversion } \\
\text { Conscientiousness }\end{array}$ & GPA & 75 & 20.8 & 72.0 & Psychology & Bachelor & $\begin{array}{r}-.37 \\
.13 \\
.29\end{array}$ \\
\hline & & $\begin{array}{l}\text { Neuroticism } \\
\text { Extraversion } \\
\text { Openness } \\
\text { Agreeableness } \\
\text { Conscientiousness }\end{array}$ & GPA & 70 & 19.8 & 70.0 & Psychology & Bachelor & $\begin{array}{r}-.35 \\
.07 \\
.22 \\
.00 \\
.39\end{array}$ \\
\hline $\begin{array}{l}\text { Chamorro-Prem- } \\
\text { muzic \& Furnham } \\
\text { (2003b) }\end{array}$ & UK & $\begin{array}{l}\text { Neuroticism } \\
\text { Extraversion } \\
\text { Openness } \\
\text { Agreeableness } \\
\text { Conscientiousness }\end{array}$ & GPA & 274 & 20.1 & 72.5 & Psychology & Bachelor & $\begin{array}{r}-.16 \\
-.11 \\
.02 \\
.07 \\
.36\end{array}$ \\
\hline $\begin{array}{l}\text { Colquitt \& Sim- } \\
\text { mering (1998) }\end{array}$ & USA & Conscientiousness & GPA & 103 & - & - & Economics & Bachelor & .24 \\
\hline
\end{tabular}




\begin{tabular}{|c|c|c|c|c|c|c|c|c|c|}
\hline Author(s) & Country & Predictor & Criterion & $N$ & $A$ & $F$ & Study major & Study level & $r$ \\
\hline \multirow[t]{2}{*}{$\begin{array}{l}\text { De Fruyt \& } \\
\text { Mervielde (1996) }\end{array}$} & \multirow[t]{2}{*}{ Belgium } & $\begin{array}{l}\text { Neuroticism } \\
\text { Extraversion } \\
\text { Openness } \\
\text { Agreeableness } \\
\text { Conscientiousness }\end{array}$ & GPA & 343 & 23.4 & .0 & Multiple majors & Main study period & $\begin{array}{r}-.18 \\
.05 \\
-.11 \\
.06 \\
.35\end{array}$ \\
\hline & & $\begin{array}{l}\text { Neuroticism } \\
\text { Extraversion } \\
\text { Openness } \\
\text { Agreeableness } \\
\text { Conscientiousness }\end{array}$ & GPA & 397 & 23.4 & 100.0 & Multiple majors & Main study period & $\begin{array}{r}-.04 \\
-.02 \\
-.10 \\
.00 \\
.22\end{array}$ \\
\hline \multirow[t]{2}{*}{ Diseth (2003) } & \multirow[t]{2}{*}{ Norway } & $\begin{array}{l}\text { Neuroticism } \\
\text { Extraversion } \\
\text { Openness } \\
\text { Agreeableness } \\
\text { Conscientiousness }\end{array}$ & $\begin{array}{l}\text { \#Individual } \\
\text { score }\end{array}$ & 127 & 21.4 & 77.0 & Psychology & Basic study period & $\begin{array}{r}-.03 \\
-.10 \\
.03 \\
.12 \\
.06\end{array}$ \\
\hline & & $\begin{array}{l}\text { Neuroticism } \\
\text { Extraversion } \\
\text { Openness } \\
\text { Agreeableness } \\
\text { Conscientiousness }\end{array}$ & $\begin{array}{l}\text { Individual } \\
\text { score }\end{array}$ & 101 & 20.2 & 64.0 & $\begin{array}{l}\text { Linguistics and cul- } \\
\text { tural sciences }\end{array}$ & Basic study period & $\begin{array}{r}.20 \\
-.07 \\
.22 \\
-.21 \\
-.10\end{array}$ \\
\hline $\begin{array}{l}\text { Dollinger \& Orf } \\
\text { (1991) }\end{array}$ & USA & $\begin{array}{l}\text { Neuroticism } \\
\text { Extraversion } \\
\text { Openness } \\
\text { Agreeableness } \\
\text { Conscientiousness }\end{array}$ & $\begin{array}{l}\text { Individual } \\
\text { score }\end{array}$ & 90 & - & 73.3 & Psychology & Bachelor & $\begin{array}{r}-.01 \\
.11 \\
.20 \\
.05 \\
.25\end{array}$ \\
\hline $\begin{array}{l}\text { Duff, Boyle, } \\
\text { Dunleavy, \& } \\
\text { Ferguson (2004) }\end{array}$ & UK & $\begin{array}{l}\text { Neuroticism } \\
\text { Extraversion } \\
\text { Openness } \\
\text { Agreeableness } \\
\text { Conscientiousness }\end{array}$ & GPA & 146 & 24.3 & 74.7 & Social sciences & Bachelor & $\begin{array}{r}-.14 \\
.06 \\
.07 \\
.12 \\
.21\end{array}$ \\
\hline Dyer (1987) & USA & Openness & GPA & 868 & - & - & Medical sciences & Bachelor & .10 \\
\hline $\begin{array}{l}\text { Edwards \& } \\
\text { Schleicher (2004) }\end{array}$ & USA & $\begin{array}{l}\text { Neuroticism } \\
\text { Agreeableness }\end{array}$ & GPA & 70 & - & 61.4 & Psychology & Master & $\begin{array}{l}.12 \\
.02\end{array}$ \\
\hline $\begin{array}{l}\text { Farsides \& } \\
\text { Woodfield (2003) }\end{array}$ & UK & $\begin{array}{l}\text { Neuroticism } \\
\text { Extraversion } \\
\text { Openness } \\
\text { Agreeableness } \\
\text { Conscientiousness }\end{array}$ & GPA & 432 & 21.3 & 52.2 & Multiple majors & Basic study period & $\begin{array}{l}.03 \\
.00 \\
.26 \\
.14 \\
.09\end{array}$ \\
\hline $\begin{array}{l}\text { Ferguson, James, } \\
\text { O'Hehir, \& } \\
\text { Sanders (2003) }\end{array}$ & UK & $\begin{array}{l}\text { Neuroticism } \\
\text { Extraversion } \\
\text { Openness } \\
\text { Agreeableness } \\
\text { Conscientiousness }\end{array}$ & GPA & 118 & 19.7 & 58.0 & Human medicine & $\begin{array}{l}\text { Preclinical and clin- } \\
\text { ical study period }\end{array}$ & $\begin{array}{r}.07 \\
-.03 \\
.00 \\
.13 \\
26\end{array}$ \\
\hline $\begin{array}{l}\text { Furnham \& Cha- } \\
\text { morro-Premuzic } \\
\text { (2004) }\end{array}$ & UK & $\begin{array}{l}\text { Neuroticism } \\
\text { Extraversion } \\
\text { Openness } \\
\text { Agreeableness } \\
\text { Conscientiousness }\end{array}$ & $\begin{array}{l}\text { Individual } \\
\text { score }\end{array}$ & 91 & 19.7 & 81.3 & Mathematics & Bachelor & $\begin{array}{r}.05 \\
-.26 \\
-.07 \\
-.04 \\
.27\end{array}$ \\
\hline $\begin{array}{l}\text { Furnham, Chamor- } \\
\text { ro-Premuzic, \& } \\
\text { McDougall (2002) }\end{array}$ & UK & $\begin{array}{l}\text { Neuroticism } \\
\text { Extraversion } \\
\text { Openness } \\
\text { Agreeableness } \\
\text { Conscientiousness }\end{array}$ & GPA & 93 & 19.3 & 75.3 & Multiple majors & Basic study period & $\begin{array}{r}.14 \\
-.29 \\
-.16 \\
.06 \\
.40\end{array}$ \\
\hline $\begin{array}{l}\text { Furnham \& } \\
\text { Mitchell (1991) }\end{array}$ & UK & $\begin{array}{l}\text { Neuroticism } \\
\text { Extraversion } \\
\text { Conscientiousness }\end{array}$ & GPA & 252 & - & 95.0 & Medical sciences & $\begin{array}{l}\text { Preclinical study } \\
\text { period }\end{array}$ & $\begin{array}{l}-.09 \\
-.07 \\
-.05\end{array}$ \\
\hline
\end{tabular}




\begin{tabular}{|c|c|c|c|c|c|c|c|c|c|}
\hline Author(s) & Country & Predictor & Criterion & $N$ & $A$ & $F$ & Study major & Study level & $r$ \\
\hline \multirow[t]{4}{*}{ Gakhar (1986) } & \multirow[t]{4}{*}{ India } & $\begin{array}{l}\text { Neuroticism } \\
\text { Extraversion } \\
\text { Openness }\end{array}$ & GPA & 50 & - & - & $\begin{array}{l}\text { Mathematics and } \\
\text { natural sciences }\end{array}$ & Basic study period & $\begin{array}{r}-.24 \\
.19 \\
-.16\end{array}$ \\
\hline & & $\begin{array}{l}\text { Neuroticism } \\
\text { Extraversion } \\
\text { Openness }\end{array}$ & GPA & 50 & & & Economics & Basic study period & $\begin{array}{r}-.32 \\
.37 \\
.11\end{array}$ \\
\hline & & $\begin{array}{l}\text { Neuroticism } \\
\text { Extraversion } \\
\text { Openness }\end{array}$ & GPA & 50 & & & Multiple majors & Basic study period & $\begin{array}{l}-.25 \\
-.07 \\
-.21\end{array}$ \\
\hline & & $\begin{array}{l}\text { Neuroticism } \\
\text { Extraversion } \\
\text { Openness }\end{array}$ & GPA & 50 & & & Nutrition science & Basic study period & $\begin{array}{r}-.22 \\
.47 \\
.42\end{array}$ \\
\hline \multirow{19}{*}{$\begin{array}{l}\text { Giesen, Gold, } \\
\text { Hummer, \& } \\
\text { Jansen (1986) }\end{array}$} & \multirow[t]{19}{*}{ Germany } & Neuroticism & Satisfaction & 137 & - & - & Medical sciences & $\begin{array}{l}\text { Preclinical study } \\
\text { period }\end{array}$ & -.31 \\
\hline & & Extraversion & Satisfaction & 137 & & & Medical sciences & & .15 \\
\hline & & Neuroticism & Satisfaction & 141 & & & $\begin{array}{l}\text { Mathematics and } \\
\text { natural sciences }\end{array}$ & Basic study period & -.30 \\
\hline & & Extraversion & GPA & 130 & & & $\begin{array}{l}\text { Mathematics and } \\
\text { natural sciences }\end{array}$ & & -.32 \\
\hline & & Extraversion & Satisfaction & 141 & & & $\begin{array}{l}\text { Mathematics and } \\
\text { natural sciences }\end{array}$ & & -.26 \\
\hline & & Neuroticism & Satisfaction & 176 & & & $\begin{array}{l}\text { Mathematics and } \\
\text { natural sciences }\end{array}$ & & -.29 \\
\hline & & Extraversion & Satisfaction & 176 & & & $\begin{array}{l}\text { Mathematics and } \\
\text { natural sciences }\end{array}$ & & .17 \\
\hline & & Neuroticism & Satisfaction & 164 & & & Engineering & & -.27 \\
\hline & & Extraversion & GPA & 147 & & & Engineering & & -.19 \\
\hline & & Neuroticism & Satisfaction & 157 & & & Economics & -.26 & \\
\hline & & Extraversion & GPA & 113 & & & Economics & -.21 & \\
\hline & & Neuroticism & GPA & 89 & & & Law & Main study period & -.06 \\
\hline & & Neuroticism & Satisfaction & 209 & & & Law & & -.26 \\
\hline & & Extraversion & Satisfaction & 206 & & & Law & .17 & \\
\hline & & Neuroticism & GPA & 220 & & & $\begin{array}{l}\text { Linguistics and } \\
\text { cultural sciences }\end{array}$ & $\begin{array}{l}\text { Basic and main } \\
\text { study period }\end{array}$ & .19 \\
\hline & & Neuroticism & Satisfaction & 357 & & & $\begin{array}{l}\text { Linguistics and } \\
\text { cultural sciences }\end{array}$ & & -.26 \\
\hline & & Neuroticism & Satisfaction & 171 & & & Teaching profession & Main study period & -.17 \\
\hline & & Neuroticism & Retention & 1722 & & & Multiple majors & $\begin{array}{l}\text { Basic and main } \\
\text { study period }\end{array}$ & -.10 \\
\hline & & Extraversion & Retention & 1722 & & & Multiple majors & & -.06 \\
\hline $\begin{array}{l}\text { Goff \& Ackermann } \\
\text { (1992) }\end{array}$ & USA & $\begin{array}{l}\text { Neuroticism } \\
\text { Extraversion } \\
\text { Openness } \\
\text { Agreeableness } \\
\text { Conscientiousness }\end{array}$ & GPA & 138 & - & 55.8 & Multiple majors & Bachelor & $\begin{array}{r}-.09 \\
-.17 \\
.00 \\
.03 \\
.17\end{array}$ \\
\hline
\end{tabular}




\begin{tabular}{|c|c|c|c|c|c|c|c|c|c|}
\hline Author(s) & Country & Predictor & Criterion & $N$ & $A$ & $F$ & Study major & Study level & $r$ \\
\hline \multirow[t]{4}{*}{$\begin{array}{l}\text { Gough \& Lanning } \\
\text { (1986) }\end{array}$} & USA & $\begin{array}{l}\text { Neuroticism } \\
\text { Openness } \\
\text { Agreeableness }\end{array}$ & $\begin{array}{l}\text { Individual } \\
\text { score }\end{array}$ & 1347 & - & .0 & Multiple majors & Bachelor & $\begin{array}{l}.02 \\
.17 \\
.04\end{array}$ \\
\hline & & $\begin{array}{l}\text { Neuroticism } \\
\text { Openness } \\
\text { Agreeableness }\end{array}$ & & 1842 & & 100.0 & & & $\begin{array}{r}.07 \\
.15 \\
-.02\end{array}$ \\
\hline & & $\begin{array}{l}\text { Neuroticism } \\
\text { Openness } \\
\text { Agreeableness }\end{array}$ & & 326 & & .0 & Psychology & & $\begin{array}{r}-.04 \\
.25 \\
-.01\end{array}$ \\
\hline & & $\begin{array}{l}\text { Neuroticism } \\
\text { Openness } \\
\text { Agreeableness }\end{array}$ & & 570 & & 100.0 & & & $\begin{array}{r}.07 \\
.24 \\
-.06\end{array}$ \\
\hline $\begin{array}{l}\text { Gray \& Watson } \\
(2002)\end{array}$ & USA & $\begin{array}{l}\text { Neuroticism } \\
\text { Extraversion } \\
\text { Openness } \\
\text { Agreeableness } \\
\text { Conscientiousness }\end{array}$ & GPA & 334 & 19.2 & 63.8 & Multiple majors & Bachelor & $\begin{array}{r}.00 \\
-.09 \\
.18 \\
.15 \\
.36\end{array}$ \\
\hline $\begin{array}{l}\text { Hörschgen, Cierp- } \\
\text { ka, Friese, \& Stein- } \\
\text { bach (1993) }\end{array}$ & Germany & Extraversion & GPA & 371 & - & 22.5 & Economics & $\begin{array}{l}\text { Basic and main } \\
\text { study period }\end{array}$ & .19 \\
\hline \multirow[t]{2}{*}{$\begin{array}{l}\text { Huq, Rabman, \& } \\
\text { Mahmud (1986) }\end{array}$} & Bangladesh & $\begin{array}{l}\text { Neuroticism } \\
\text { Extraversion } \\
\text { Conscientiousness }\end{array}$ & GPA & 22 & - & .0 & Psychology & Bachelor & $\begin{array}{r}-.23 \\
.78 \\
.25\end{array}$ \\
\hline & & $\begin{array}{l}\text { Neuroticism } \\
\text { Extraversion } \\
\text { Conscientiousness }\end{array}$ & & 21 & & 100.0 & & & $\begin{array}{r}-.16 \\
.29 \\
.14\end{array}$ \\
\hline \multirow{3}{*}{$\begin{array}{l}\text { Kahn, Nauta, Gail- } \\
\text { breath, Tipps, \& } \\
\text { Chartrand (2002) }\end{array}$} & USA & Neuroticism & $\begin{array}{l}\text { GPA } \\
\text { Retention }\end{array}$ & 586 & 18.2 & 52.4 & Multiple majors & Bachelor & $\begin{array}{l}.16 \\
.08\end{array}$ \\
\hline & & Extraversion & $\begin{array}{l}\text { GPA } \\
\text { Retention }\end{array}$ & 674 & 18.2 & 52.4 & & & $\begin{array}{r}-.05 \\
.04\end{array}$ \\
\hline & & Openness & $\begin{array}{l}\text { GPA } \\
\text { Retention }\end{array}$ & & & & & & $\begin{array}{l}.06 \\
.05\end{array}$ \\
\hline Kalsbeek (2003) & USA & Extraversion & GPA & 989 & - & - & Multiple majors & Bachelor & -.24 \\
\hline $\begin{array}{l}\text { King \& Bailly } \\
(2002)\end{array}$ & USA & $\begin{array}{l}\text { Extraversion } \\
\text { Extraversion }\end{array}$ & $\begin{array}{l}\text { GPA } \\
\text { GPA }\end{array}$ & $\begin{array}{l}326 \\
109\end{array}$ & - & $\begin{array}{r}100.0 \\
.0\end{array}$ & Psychology & Bachelor & $\begin{array}{r}.01 \\
-.11\end{array}$ \\
\hline $\begin{array}{l}\text { Lipton, Huxham, \& } \\
\text { Hamilton (1984) }\end{array}$ & Australia & $\begin{array}{l}\text { Neuroticism } \\
\text { Extraversion } \\
\text { Conscientiousness }\end{array}$ & $\begin{array}{l}\text { Individual } \\
\text { score }\end{array}$ & 151 & - & - & Human medicine & $\begin{array}{l}\text { Preclinical and clin- } \\
\text { ical study period }\end{array}$ & $\begin{array}{r}-.12 \\
-.28 \\
.15\end{array}$ \\
\hline $\begin{array}{l}\text { Lounsbury, Sund- } \\
\text { strom, Loveland, \& } \\
\text { Gibson (2003) }\end{array}$ & USA & $\begin{array}{l}\text { Neuroticism } \\
\text { Extraversion } \\
\text { Openness } \\
\text { Agreeableness } \\
\text { Conscientiousness }\end{array}$ & $\begin{array}{l}\text { Individual } \\
\text { score }\end{array}$ & 175 & - & 64.0 & Psychology & Bachelor & $\begin{array}{r}-.11 \\
.01 \\
.16 \\
-.01 \\
.18\end{array}$ \\
\hline $\begin{array}{l}\text { Lufi, Parish-Plass, } \\
\& \text { Cohen (2003) }\end{array}$ & Israel & $\begin{array}{l}\text { Neuroticism } \\
\text { Extraversion } \\
\text { Openness } \\
\text { Agreeableness }\end{array}$ & Retention & 166 & 23.37 & 80.1 & Teaching profession & Bachelor & $\begin{array}{r}-.05 \\
.01 \\
-.13 \\
.12\end{array}$ \\
\hline $\begin{array}{l}\text { McCabe, Blank- } \\
\text { stein, \& Mills }\end{array}$ & Canada & Neuroticism & GPA & $\begin{array}{r}124 \\
66\end{array}$ & $\begin{array}{l}22.4 \\
22.4\end{array}$ & $\begin{array}{r}100.0 \\
.0\end{array}$ & Psychology & Bachelor & $\begin{array}{r}.01 \\
-.09\end{array}$ \\
\hline
\end{tabular}




\begin{tabular}{|c|c|c|c|c|c|c|c|c|c|}
\hline Author(s) & Country & Predictor & Criterion & $N$ & $A$ & $F$ & Study major & Study level & $r$ \\
\hline $\begin{array}{l}\text { McIlroy \& Bunting } \\
\text { (2002) }\end{array}$ & $\begin{array}{l}\text { Northern } \\
\text { Ireland }\end{array}$ & Conscientiousness & GPA & 219 & - & 83.1 & Psychology & Basic study period & .35 \\
\hline McKenzie (1989) & UK & $\begin{array}{l}\text { Neuroticism } \\
\text { Extraversion }\end{array}$ & GPA & 204 & - & 54.9 & Multiple majors & Bachelor & $\begin{array}{r}.17 \\
-.01\end{array}$ \\
\hline $\begin{array}{l}\text { McKenzie \& Gow } \\
(2004)\end{array}$ & Australia & $\begin{array}{l}\text { Neuroticism } \\
\text { Extraversion } \\
\text { Openness } \\
\text { Agreeableness } \\
\text { Conscientiousness }\end{array}$ & GPA & 1193 & 21.44 & 51.8 & Multiple majors & Bachelor & $\begin{array}{r}-.02 \\
-.05 \\
.09 \\
.09 \\
.18\end{array}$ \\
\hline $\begin{array}{l}\text { McKenzie, Tag- } \\
\text { havi-Khonsary, \& } \\
\text { Tindell (2000) }\end{array}$ & UK & $\begin{array}{l}\text { Neuroticism } \\
\text { Extraversion }\end{array}$ & GPA & 67 & - & - & Multiple majors & Bachelor & $\begin{array}{r}.13 \\
-.05\end{array}$ \\
\hline $\begin{array}{l}\text { McKenzie \& } \\
\text { Tindell (1993) }\end{array}$ & UK & $\begin{array}{l}\text { Neuroticism } \\
\text { Extraversion }\end{array}$ & GPA & 60 & 27.73 & - & Multiple majors & Bachelor & $\begin{array}{l}.02 \\
.09\end{array}$ \\
\hline $\begin{array}{l}\text { Mehta \& Kumar } \\
\text { (1985) }\end{array}$ & India & $\begin{array}{l}\text { Neuroticism } \\
\text { Extraversion }\end{array}$ & GPA & 120 & - & 50.0 & Multiple majors & $\begin{array}{l}\text { Bachelor and } \\
\text { master }\end{array}$ & $\begin{array}{r}.00 \\
-.01\end{array}$ \\
\hline $\begin{array}{l}\text { Moon \& Illing- } \\
\text { worth (2005) }\end{array}$ & USA & $\begin{array}{l}\text { Neuroticism } \\
\text { Conscientiousness }\end{array}$ & GPA & 303 & 21.89 & 64.0 & Psychology & Bachelor & $\begin{array}{r}-.08 \\
.05\end{array}$ \\
\hline $\begin{array}{l}\text { Morris \& Carden } \\
\text { (1981) }\end{array}$ & USA & $\begin{array}{l}\text { Neuroticism } \\
\text { Extraversion }\end{array}$ & $\begin{array}{l}\text { Individual } \\
\text { score }\end{array}$ & 58 & - & - & Psychology & Bachelor & $\begin{array}{l}-.13 \\
-.06\end{array}$ \\
\hline $\begin{array}{l}\text { Musgrave-Mar- } \\
\text { quart, Bromley, } \\
\& \text { Dalley (1997) }\end{array}$ & USA & $\begin{array}{l}\text { Neuroticism } \\
\text { Extraversion } \\
\text { Openness } \\
\text { Agreeableness } \\
\text { Conscientiousness }\end{array}$ & GPA & 161 & 20.8 & 44.0 & Psychology & Bachelor & $\begin{array}{r}.22 \\
-.02 \\
.24 \\
.25 \\
.32\end{array}$ \\
\hline Nath (1987) & India & $\begin{array}{l}\text { Neuroticism } \\
\text { Extraversion }\end{array}$ & $\begin{array}{l}\text { Individual } \\
\text { score }\end{array}$ & 155 & - & .0 & Human medicine & $\begin{array}{l}\text { Preclinical study } \\
\text { period }\end{array}$ & $\begin{array}{r}-.32 \\
.45\end{array}$ \\
\hline \multirow[t]{5}{*}{$\begin{array}{l}\text { Okun \& Finch } \\
\text { (1998) }\end{array}$} & USA & Neuroticism & $\begin{array}{l}\text { GPA } \\
\text { Retention }\end{array}$ & 240 & 18.0 & 84.0 & Multiple majors & Bachelor & $\begin{array}{r}.04 \\
-.01\end{array}$ \\
\hline & & Extraversion & $\begin{array}{l}\text { GPA } \\
\text { Retention }\end{array}$ & & & & & & $\begin{array}{r}-.03 \\
.00\end{array}$ \\
\hline & & Openness & $\begin{array}{l}\text { GPA } \\
\text { Retention }\end{array}$ & & & & & & $\begin{array}{l}-.09 \\
-.03\end{array}$ \\
\hline & & Agreeableness & $\begin{array}{l}\text { GPA } \\
\text { Retention }\end{array}$ & & & & & & $\begin{array}{l}.05 \\
.11\end{array}$ \\
\hline & & Conscientiousness & $\begin{array}{l}\text { GPA } \\
\text { Retention }\end{array}$ & & & & & & $\begin{array}{l}.20 \\
.12\end{array}$ \\
\hline Oosthuizen (1990) & South Africa & Neuroticism & GPA & 69 & - & - & Psychology & Bachelor & .06 \\
\hline $\begin{array}{l}\text { Oswald, Schmitt, } \\
\text { Kim, Ramsay, \& } \\
\text { Gillespie (2004) }\end{array}$ & USA & $\begin{array}{l}\text { Neuroticism } \\
\text { Extraversion } \\
\text { Openness } \\
\text { Agreeableness } \\
\text { Conscientiousness }\end{array}$ & GPA & 611 & 18.5 & 72.0 & Multiple majors & Bachelor & $\begin{array}{r}-.07 \\
-.03 \\
.03 \\
.10 \\
.21\end{array}$ \\
\hline $\begin{array}{l}\text { Paunonen \& } \\
\text { Ashton (2001) }\end{array}$ & Canada & $\begin{array}{l}\text { Openness } \\
\text { Conscientiousness }\end{array}$ & $\begin{array}{l}\text { Individual } \\
\text { score }\end{array}$ & 717 & - & 73.5 & Psychology & Bachelor & $\begin{array}{r}-.04 \\
.21\end{array}$ \\
\hline $\begin{array}{l}\text { Peeters \& Lievens } \\
\text { (2005) }\end{array}$ & Belgium & $\begin{array}{l}\text { Neuroticism } \\
\text { Extraversion } \\
\text { Openness } \\
\text { Agreeableness } \\
\text { Conscientiousness }\end{array}$ & GPA & 293 & 20.6 & 81.0 & Psychology & Basic study period & $\begin{array}{r}-.03 \\
.03 \\
.07 \\
.08 \\
.28\end{array}$ \\
\hline
\end{tabular}




\begin{tabular}{|c|c|c|c|c|c|c|c|c|c|}
\hline Author(s) & Country & Predictor & Criterion & $N$ & $A$ & $F$ & Study major & Study level & $r$ \\
\hline $\begin{array}{l}\text { Peng, Khaw, \& } \\
\text { Edariah (1995) }\end{array}$ & Malaysia & $\begin{array}{l}\text { Neuroticism } \\
\text { Extraversion } \\
\text { Openness }\end{array}$ & GPA & 101 & 20.8 & 53.5 & Human medicine & $\begin{array}{l}\text { Preclinical study } \\
\text { period }\end{array}$ & $\begin{array}{l}.03 \\
.49 \\
.37\end{array}$ \\
\hline $\begin{array}{l}\text { Ridgell \& Louns- } \\
\text { bury (2004) }\end{array}$ & USA & $\begin{array}{l}\text { Neuroticism } \\
\text { Extraversion } \\
\text { Openness } \\
\text { Agreeableness } \\
\text { Conscientiousness }\end{array}$ & GPA & 140 & 19.2 & 46.0 & Psychology & Bachelor & $\begin{array}{r}-.13 \\
.04 \\
.06 \\
.10 \\
.15\end{array}$ \\
\hline \multirow[t]{2}{*}{$\begin{array}{l}\text { Rothstein, Pauno- } \\
\text { nen, Rush, \& King } \\
\text { (1994) }\end{array}$} & Canada & $\begin{array}{l}\text { Neuroticism } \\
\text { Extraversion } \\
\text { Openness } \\
\text { Agreeableness } \\
\text { Conscientiousness }\end{array}$ & GPA & 225 & - & 19.6 & Economics & Master & $\begin{array}{r}-.08 \\
.05 \\
.17 \\
-.16 \\
.14\end{array}$ \\
\hline & & $\begin{array}{l}\text { Neuroticism } \\
\text { Extraversion } \\
\text { Openness } \\
\text { Agreeableness } \\
\text { Conscientiousness }\end{array}$ & & 225 & & 21.8 & Economics & Master & $\begin{array}{r}-.08 \\
.09 \\
.05 \\
-.23 \\
.04\end{array}$ \\
\hline $\begin{array}{l}\text { Schmidt-Atzert } \\
(2005)\end{array}$ & Germany & Neuroticism & GPA & 48 & - & - & Psychology & Basic study period & .17 \\
\hline $\begin{array}{l}\text { Schurr, Ruble, Pa- } \\
\text { lomba, Pickerill, \& } \\
\text { Moore (1997) }\end{array}$ & USA & $\begin{array}{l}\text { Extraversion } \\
\text { Openness }\end{array}$ & Retention & 1114 & - & 62.0 & Multiple majors & Master & $\begin{array}{l}.02 \\
.05\end{array}$ \\
\hline $\begin{array}{l}\text { Tross, Harper, } \\
\text { Osher, \& Kneidin- } \\
\text { ger }(2000)\end{array}$ & USA & Conscientiousness & $\begin{array}{l}\text { GPA } \\
\text { Retention }\end{array}$ & 844 & - & 29.5 & Psychology & Bachelor & $\begin{array}{l}.35 \\
.16\end{array}$ \\
\hline Tutton (1996) & Australia & $\begin{array}{l}\text { Neuroticism } \\
\text { Openness } \\
\text { Agreeableness }\end{array}$ & GPA & 133 & - & - & Human medicine & $\begin{array}{l}\text { Preclinical study } \\
\text { period }\end{array}$ & $\begin{array}{r}.02 \\
-.04 \\
.07\end{array}$ \\
\hline $\begin{array}{l}\text { Upmanyu, Upman- } \\
\text { yu, \& Vasudeva } \\
\text { (1980) }\end{array}$ & India & $\begin{array}{l}\text { Neuroticism } \\
\text { Extraversion } \\
\text { Conscientiousness }\end{array}$ & GPA & 100 & 20.9 & .0 & Social sciences & - & $\begin{array}{r}-.26 \\
.05 \\
.04\end{array}$ \\
\hline $\begin{array}{l}\text { Watkins \& Astilla } \\
\text { (1980) }\end{array}$ & Philippines & $\begin{array}{l}\text { Openness } \\
\text { Agreeableness }\end{array}$ & GPA & 1149 & - & 73.3 & Multiple majors & Bachelor & $\begin{array}{l}.14 \\
.11\end{array}$ \\
\hline \multirow[t]{2}{*}{$\begin{array}{l}\text { Watson \& Clark } \\
\text { (1993) }\end{array}$} & USA & $\begin{array}{l}\text { Neuroticism } \\
\text { Extraversion } \\
\text { Conscientiousness }\end{array}$ & GPA & 259 & - & - & Multiple majors & Bachelor & $\begin{array}{l}.01 \\
.10 \\
.34\end{array}$ \\
\hline & & Conscientiousness & & 69 & & & & & .37 \\
\hline $\begin{array}{l}\text { Wolfe \& Johnson } \\
\text { (1995) }\end{array}$ & USA & $\begin{array}{l}\text { Neuroticism } \\
\text { Extraversion } \\
\text { Openness } \\
\text { Agreeableness } \\
\text { Conscientiousness }\end{array}$ & GPA & 201 & - & 78.1 & Psychology & Bachelor & $\begin{array}{r}-.02 \\
-.08 \\
.10 \\
.08 \\
.34\end{array}$ \\
\hline
\end{tabular}

Notes. $N=$ sample size; $A=$ sample mean age (years); $F=$ percent female students in the sample; $r=$ uncorrected product-moment correlation (raw, pooled, or averaged); GPA: Grade point average.

58 articles and reports from 15 different countries (Australia, Austria, Bangladesh, Belgium, Canada, Germany, India, Israel, Malaysia, Netherlands, Norway, Philippines, South Africa, UK, and US) were used for meta-analytic calculations. With respect to the success criterion grades, more than 30 validity coefficients from independent samples were found for each of the five personality traits. Concerning the criteria retention and academic satisfaction, we obtained at least three coefficients for Neuroticism, Extraversion, and Openness to Experience. This allowed us to calculate meta-analyses on these factors. The number of validity coefficients and sample sizes for each predictor and each criterion is presented in Table 1. Table 2 shows all primary studies included.

All studies included report self-ratings of personality traits, measured with different instruments in different lan- 
guages and editions. Frequently used instruments were, for example: NEO-PI-R (Costa \& McCrae, 1992b), EPI (Eysenck Personality Inventory; Eysenck \& Eysenck, 1965), EPQ-R (Eysenck Personality Questionnaire; Eysenck, \& Eysenck, 1975), 16PF (Sixteen Personality Factor Questionnaire; e.g., Cattell, Eber, \& Tatsuoka, 1970), CPI (California Psychological Inventory; Gough, 1975), IPIP (International Personality Item Pool; Goldberg et al., 2006), and NEO-FFI (Costa \& McCrae, 1991). Personality was measured in a voluntary context (usually within the scope of a course) in all studies. None of the included studies provided information about personality measures under selection conditions.

\section{Meta-Analytic Approach}

The collected data were analyzed using the validation generalization approach of Raju, Burke, Normand, and Langlois (1991), which is based on the meta-analytic method of Hunter, Schmidt, and Jackson (1982). We used the corresponding computer program of Raju and Fleer (2003) to calculate meta-analyses under the random-effects (RE) and fixed-effects (FE) conditions. With respect to subject matter, the assumptions made by the RE model are more adequate than the assumptions made by the FE model. The FE model postulates a single true effect with all variance between studies caused by sample error, measurement error or other adulterant or moderating influences. The RE model deals with a distribution of true effects. In the RE model the true effect varies across situations and, hence, has true variance. Meta-analyses under RE model conditions calculate the mean true effect. According to Schulze (2004), estimations under the RE model assumption are not reliable until at least 32 coefficients are included. Therefore, we report the results of both the RE and the FE model. The mean correlation calculated under the FE or RE conditions is the same, but confidence intervals differ. The assumptions of the FE model lead to smaller confidence-intervals, so that both effects and moderator variables are more likely to become significant In accordance with Hunter and Schmidt (2004, p. 83), Fisher's z-transformation was not used.

\section{Procedure}

Studies were included if they reported correlations (or statistics convertible to correlations) between one or more of the Big Five personality factors and at least one outcome measure of university success (GPA, retention, and/or satisfaction). The classification of scales from inventories into the five factors was mainly based on the classifications made by Hough and Ones (2002). For example, "Achievement (N Ach)" of the Manifest Needs Questionnaire (MNQ; Steers \& Braunstein, 1976) was coded as the fourth facet of Conscientiousness (achievement striving). Further- more, scales were categorized using data and descriptions given in the test manuals (e.g., Rossier, Meyer de Stadelhofen, \& Berthoud, 2004; Ostendorf \& Angleitner, 2004; Fleenor \& Eastman, 1997; Gough, 1975). In one report the classification had already been done by the authors themselves (Brandstätter \& Farthofer, 2002). In the rare cases where no satisfying empirical information was found but the study provided detailed scale descriptions, we decided upon classifications by consensus.

Meta-analyses were calculated on a basis of at least three coefficients. Just one coefficient was analyzed for each sample and predictor-criterion combination and 79 details were coded for each coefficient. When studies reported two or more coefficients for the same relationship and sample, these correlations were pooled with regard to their intercorrelation. The reliability of the pooled predictors was estimated with Mosier's formula (Hunter \& Schmidt, 2004). For this purpose we used the program of Schmidt and Le (2004). When the intercorrelations were unknown, coefficients were averaged.

The following aspects have been considered in the coding process: general aspects (e.g., country of origin, publication date), sample characteristics (e.g., size, ethnicity, age), predictor characteristics (e.g., trait, instrument, reliability), criterion characteristics (e.g., kind of criterion, reliability), validity characteristics (e.g., applied statistic), and moderator variables (e.g., study major, study period, time between measurement of predictor and criterion).

\section{Consideration of Artifacts}

Validity coefficients were corrected for sampling error and attenuation by error of measurement in predictors and criteria. The studies yielded little information about reliabilities. Measurement error of predictors was corrected using retest-reliabilities reported in the article or in the test manual of the instrument used. Otherwise we used the reliabilities provided by Viswesvaran and Ones (2000). To correct measurement error of the grades criterion, we used the reliability estimation of GPA provided by Kuncel et al. (2004) who calculated an overall reliability of $r_{t t}=.83$. Some studies reported only correlations based on individual grades. Empirical studies estimate the reliability of single university grades at .55 to .70 for oral examinations in psychology (Novy, Kopel, \& Swank, 1996) and .65 for oral examinations in medicine (Wass, Wakeford, Neighbor, \& van der Vleuten, 2003). Reliabilities for written examinations are probably higher. We calculated reliability for single grades with the Spearman-Brown prophecy formula based on the assumption that GPA with reliability of .83 consists of at least two scores. The result was a reliability estimate of $r_{t t}=.71$. This is a rather optimistic assumption for individual scores, which leads to conservative corrections of the validity but seems adequate when thinking of written examinations as more common than oral examinations. The estimated reliability of satisfaction scales is .83 
Table 3. Results of the meta-analysis for the Big Five personality traits and the criterion grades

\begin{tabular}{|c|c|c|c|c|c|c|c|c|c|c|c|}
\hline & $N$ & $k$ & $M$ & $\hat{\mu}_{\rho}$ & $\hat{\sigma}_{\rho}^{2}$ & $\%$ & $S E_{F E}$ & $95 \% C I_{F E}$ & $S E_{R E}$ & $95 \% C I_{R E}$ & $90 \% \mathrm{CV}$ \\
\hline Neuroticism & 14653 & 59 & -.044 & $\begin{array}{l}-.003 \\
(-.005)\end{array}$ & $\begin{array}{l}.012 \\
(.007)\end{array}$ & $\begin{array}{c}34.8 \\
(35.2)\end{array}$ & $\begin{array}{l}.010 \\
(.008)\end{array}$ & $\begin{array}{l}{[-.024, .017]} \\
(-.021, .011)\end{array}$ & $\begin{array}{l}.018 \\
(.014)\end{array}$ & $\begin{array}{l}{[-.038, .032]} \\
(-.032, .022)\end{array}$ & $\begin{array}{l}.138 \\
(.104)\end{array}$ \\
\hline Extraversion & 12424 & 54 & .011 & $\begin{array}{l}-.059 \\
(-.047)\end{array}$ & $\begin{array}{l}.031 \\
(.018)\end{array}$ & $\begin{array}{c}17.1 \\
(18.2)\end{array}$ & $\begin{array}{l}.011 \\
(.009)\end{array}$ & $\begin{array}{l}{[-.081,-.038]} \\
(-.064,-.031)\end{array}$ & $\begin{array}{l}.026 \\
(.020)\end{array}$ & $\begin{array}{l}{[-.111,-.008]} \\
(-.087,-.008)\end{array}$ & $\begin{array}{l}.166 \\
(.125)\end{array}$ \\
\hline $\begin{array}{l}\text { Openness to } \\
\text { Experience }\end{array}$ & 14942 & 41 & .083 & $\begin{array}{l}.133 \\
(.099)\end{array}$ & $\begin{array}{l}.015 \\
(.008)\end{array}$ & $\begin{array}{c}23.3 \\
(25.2)\end{array}$ & $\begin{array}{l}.011 \\
(.008)\end{array}$ & $\begin{array}{l}{[.113, .154]} \\
(.083, .114)\end{array}$ & $\begin{array}{l}.022 \\
(.016)\end{array}$ & $\begin{array}{l}{\left[\begin{array}{l}.091, .176] \\
(.067, .130)\end{array}\right.}\end{array}$ & $\begin{array}{l}-.023 \\
(-.014)\end{array}$ \\
\hline Agreeableness & 12452 & 34 & .041 & $\begin{array}{l}.059 \\
(.042)\end{array}$ & $\begin{array}{l}.008 \\
(.004)\end{array}$ & $\begin{array}{c}41.8 \\
(42.0)\end{array}$ & $\begin{array}{l}.013 \\
(.009)\end{array}$ & $\begin{array}{l}{[.035, .084]} \\
(.025, .059)\end{array}$ & $\begin{array}{l}.019 \\
(.014)\end{array}$ & $\begin{array}{l}{\left[\begin{array}{l}.021, .098] \\
(.015, .069)\end{array}\right.}\end{array}$ & $\begin{array}{l}-.052 \\
(-.036)\end{array}$ \\
\hline Conscientiousness & 10855 & 41 & .216 & $\begin{array}{c}.269 \\
(.211)\end{array}$ & $\begin{array}{c}.014 \\
(.009)\end{array}$ & $\begin{array}{c}27.0 \\
(27.7)\end{array}$ & $\begin{array}{l}.011 \\
(.009)\end{array}$ & $\begin{array}{l}{[.247, .292]} \\
(.193, .228)\end{array}$ & $\begin{array}{c}.022 \\
(.017)\end{array}$ & 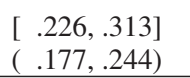 & $\begin{array}{l}.116 \\
(.091)\end{array}$ \\
\hline
\end{tabular}

Notes: $N=$ overall sample size; $k=$ number of independent samples; $M=$ mean observed correlation; $\hat{\mu}_{\rho}=$ sample size weighted and corrected validity; $\hat{\sigma}_{\rho}^{2}=$ estimated variance of $\rho ; \%=$ percent of variance accounted for by artifacts; $S E_{F E}=$ standard error of $\rho$, fixed effects model; $95 \%$ $C I_{F E}=$ confidence interval with $p=.95$, fixed effects model; $S E_{R E}=$ standard error of $\rho$, random effects model; $95 \% C I_{R E}=$ confidence interval with $p=.95$, random effects model; $90 \% C V=$ credibility value: lower limit for positive $\hat{\mu}_{\rho}$-values, upper limits for negative $\hat{\mu}_{\mathrm{p}}$-values of 90\%-credibility interval; ( ) = results not corrected for attenuation by measurement error.

Table 4. Results of the meta-analysis for the predictors Neuroticism, Extraversion, and Openness to Experience and the criterion retention

\begin{tabular}{llllllllllll}
\hline & $N$ & $k$ & $M$ & $\hat{\mu}_{\rho}$ & $\hat{\sigma}_{\rho}^{2}$ & $\%$ & $S E_{F E}$ & $95 \% C I_{F E}$ & $S E_{R E}$ & $95 \% C I_{R E}$ & $90 \% C V$ \\
\hline Neuroticism & 2714 & 4 & -.020 & -.058 & .005 & 26.5 & .022 & {$[-.101,-.015]$} & .042 & {$[-.141, .025]$} & .035 \\
& & & & $(-.050)$ & $(.004)$ & $(27.2)$ & $(.019)$ & $(-.088,-.013)$ & $(.037)$ & $(-.122, .022)$ & $(.030)$ \\
Extraversion & \multirow{2}{*}{3916} & 5 & .038 & -.015 & .001 & 69.0 & .018 & {$[-.051, .020]$} & .022 & {$[-.058, .027]$} & .019 \\
& & & & $(-.013)$ & $(.001)$ & $(71.1)$ & $(.016)$ & $(-.045, .018)$ & $(.019)$ & $(-.051, .024)$ & $(.016)$ \\
Openness to & 2194 & \multirow{2}{*}{4} & -.015 & .031 & .002 & 61.0 & .026 & {$[-.019, .082]$} & .033 & {$[-.033, .096]$} & -.021 \\
Experience & & & & $(.028)$ & $(.001)$ & $(68.2)$ & $(.021)$ & $(-.014, .069)$ & $(.026)$ & $(-.023, .078)$ & $(-.010)$ \\
\hline
\end{tabular}

Notes: see Table 3.

as reported by Judge et al. (2002). Retention can be measured without error and has, therefore, not been adjusted. Correction for range restriction was not implemented; we assumed that most of the students had not been selected by personality traits. The variance-reducing effect of students with specific traits dropping out of college, on the other hand, cannot be estimated with the current empirical data.

\section{Results}

A total of 258 correlations ( 229 correlations with the grades criterion, 13 correlations with the retention criterion, and 12 with the satisfaction criterion) were obtained from the 58 studies. Sample sizes ranged from 21 to $1,842(M=$ 301.6, $S D=343.3$ ).

Table 3 shows the meta-analytic results for the criterion college grades. Substantial validity was found for Conscientiousness $\left(\hat{\mu}_{\rho}=.269\right)$. This validity can be generalized, because the $95 \%$ confidence interval does not include zero and the lower limit of the $90 \%$ credibility interval is positive. However, only $27 \%$ of the variance is accounted for by artifacts. The other four personality traits (Neuroticism, Extraversion, Openness to Experience, and Agreeableness) offer no substantial and generalizable validity for college grades. The validity of Openness to Experience $\left(\mu_{\rho}=.133\right)$ for grades is the second highest after Conscientiousness. The confidence interval does not include zero, but the lower credibility value is negative. That means that less than 90 percent of the $\rho$ distribution is positive and there are situations in which Openness to Experience does not predict GPA at all. The positive validity of 133 for university grades cannot be generalized across situations. The percentage of variance accounted for by artifacts is also small, thus, moderator variables might be influencing this validity.

Table 4 lists the meta-analytic results for the retention criterion. The factors Neuroticism, Extraversion, and Openness to Experience do not predict retention. Both confidence intervals and credibility intervals include zero. Because of the lack of a sufficient number of observed correlations $(k<3)$, meta-analyses were not calculated for Agreeableness and Conscientiousness.

As can be seen in Table 5, Neuroticism and academic satisfaction are negatively associated $\left(\hat{\mu}_{\rho}=-.369\right)$ to a considerable extent. Extraversion is positively correlated with satisfaction $\left(\hat{\mu}_{\rho}=.102\right)$. However, this coefficient cannot be generalized, because the confidence interval includes zero and artifacts explain too little of the variance (18\%).

The relationship between retention and Agreeableness as well as the one between retention and Conscientiousness could not be calculated because not enough zero-order correlations were found. Too few coefficients were available 
Table 5. Results of the meta-analysis for the predictors Neuroticism and Extraversion and the criterion satisfaction

\begin{tabular}{|c|c|c|c|c|c|c|c|c|c|c|c|}
\hline & $N$ & $k$ & $M$ & $\hat{\mu}_{\rho}$ & $\hat{\sigma}_{\rho}^{2}$ & $\%$ & $S E_{F E}$ & $95 \% C I_{F E}$ & $S E_{R E}$ & $95 \% C I_{R E}$ & $90 \% \mathrm{CV}$ \\
\hline Neuroticism & 1512 & 8 & -.265 & $\begin{array}{l}-.369 \\
(-.263)\end{array}$ & $\begin{array}{l}.000 \\
(.000)\end{array}$ & $\begin{array}{c}100.0 \\
(100.0)\end{array}$ & $\begin{array}{l}.033 \\
(.024)\end{array}$ & $\begin{array}{l}{[-.433,-.305]} \\
(-.310,-.216)\end{array}$ & $\begin{array}{l}.019 \\
(.13)\end{array}$ & $\begin{array}{l}{[-.406,-.333]} \\
(-.289,-.237)\end{array}$ & $\begin{array}{l}-.369 \\
(-.263)\end{array}$ \\
\hline Extraversion & 660 & 4 & .058 & $\begin{array}{l}.102 \\
(.074) \\
\end{array}$ & $\begin{array}{l}.048 \\
(.025)\end{array}$ & $\begin{array}{c}18.1 \\
(18.6)\end{array}$ & $\begin{array}{c}.051 \\
(.038)\end{array}$ & $\begin{array}{l}{[.002, .203]} \\
(.000, .148)\end{array}$ & $\begin{array}{r}.120 \\
(.087)\end{array}$ & $\begin{array}{l}{[-.134, .338]} \\
(-.097, .245)\end{array}$ & $\begin{array}{l}-.177 \\
(-.127)\end{array}$ \\
\hline
\end{tabular}

Notes: see Table 3.

Table 6. Results of the meta-analysis for the predictor Extraversion and the criterion grades separated in four regions of origin of the study

\begin{tabular}{|c|c|c|c|c|c|c|c|c|c|c|c|}
\hline & $N$ & $k$ & $M$ & $\hat{\mu}_{p}$ & $\hat{\sigma}_{\rho}^{2}$ & $\%$ & $S E_{F E}$ & $95 \% C I_{F E}$ & $S E_{R E}$ & $95 \% C I_{R E}$ & $90 \% C V$ \\
\hline Australia & 1344 & 2 & -.165 & $\begin{array}{l}-.097 \\
(-.076)\end{array}$ & $\begin{array}{l}.008 \\
(.004)\end{array}$ & $\begin{array}{l}21.6 \\
(26.1)\end{array}$ & $\begin{array}{l}.033 \\
(.026)\end{array}$ & $\begin{array}{l}{[-.162,-.032]} \\
(-.127,-.024)\end{array}$ & $\begin{array}{l}.071 \\
(.051)\end{array}$ & $\begin{array}{l}{[-.237,043]} \\
(-.177, .025)\end{array}$ & $\begin{array}{l}.018 \\
(.004)\end{array}$ \\
\hline Europe & 4991 & 23 & -.065 & $\begin{array}{l}-.087 \\
(-.067)\end{array}$ & $\begin{array}{l}.024 \\
(.014)\end{array}$ & $\begin{array}{l}22.7 \\
(23.9)\end{array}$ & $\begin{array}{c}.017 \\
(.014)\end{array}$ & $\begin{array}{l}{[-.211,-.053]} \\
(-.095,-.040)\end{array}$ & $\begin{array}{c}.037 \\
(.028)\end{array}$ & $\begin{array}{l}{[-.158,-.015]} \\
(-.123,-.012)\end{array}$ & $\begin{array}{l}.111 \\
(.085)\end{array}$ \\
\hline North America & 5370 & 19 & -.031 & $\begin{array}{l}-.080 \\
(-.064)\end{array}$ & $\begin{array}{l}.012 \\
(.008)\end{array}$ & $\begin{array}{c}30.9 \\
(30.4)\end{array}$ & $\begin{array}{c}.017 \\
(.014)\end{array}$ & $\begin{array}{l}{[-.114,-.047]} \\
(-.091,-.038)\end{array}$ & $\begin{array}{l}.031 \\
(.025)\end{array}$ & $\begin{array}{l}{[-.141,-.020]} \\
(-.113,-.016)\end{array}$ & $\begin{array}{l}.063 \\
(.050)\end{array}$ \\
\hline East Asia & 719 & 10 & .301 & $\begin{array}{c}.356 \\
(.270)\end{array}$ & $\begin{array}{c}.077 \\
(.043)\end{array}$ & $\begin{array}{c}17.0 \\
(19.8)\end{array}$ & $\begin{array}{l}.040 \\
(.033)\end{array}$ & $\begin{array}{l}{[.278, .433]} \\
(.206, .334)\end{array}$ & $\begin{array}{c}.096 \\
(.073)\end{array}$ & $\begin{array}{l}{[.167, .545]} \\
(.127, .413)\end{array}$ & $\begin{array}{l}.000 \\
(.005)\end{array}$ \\
\hline
\end{tabular}

Notes: see Table 3.

for the relations between satisfaction and Openness to Experience, Agreeableness and Conscientiousness, also. Therefore, hypotheses concerning these correlations could not be checked.

\section{Moderator Analysis}

Artifacts did not explain more than $75 \%$ of the variance except for the relation between Neuroticism and the satisfaction criterion. Homogeneity tests $(Q$-statistics) were significant for all predictors of college grades $(p<.01)$. Hence, moderator analyses were indicated. Unfortunately, it was not possible to calculate moderator analyses for the retention and the satisfaction criterion because of the small number of coefficients in these cases. To detect significant moderator influences for college grades, multiple regression analyses and analyses of variance were calculated. Continuous moderator variables (e.g., sample mean age or time between measurement of predictor and criterion) were evaluated using multiple regression analyses. The impact of the moderator variables study major and country of origin were investigated by calculating analyses of variance. Moderator analyses were calculated under the assumptions of the RE model whenever at least 32 studies were included, otherwise under the assumptions of the FE model. Investigated moderator variables were: publication date, country of origin, sample mean age, time between measurement of predictor and criterion, heterogeneity of the sample concerning diverse universities, concerning starting years, or concerning study majors, number of discriminations in criterion, study period (Bachelor vs. Master, basic studies vs. main studies), publication source, and study major.

As we investigated 11 possible moderator variables, we adjusted 5\%- $\alpha$ with the Bonferroni procedure to .455\%. This significance level was only reached by two moderator variables: study period moderates the relationship between grades and Agreeableness and country of origin moderates the validity of Extraversion for college grades. Considering the moderator study period, the correlation is higher for basic studies and Bachelor grades than for main studies and Master grades $\left(k=32, R^{2}=.217, p<.00455\right)$, but not substantial (Bachelor: $\hat{\mu}_{\rho}=.072,90 \%$ lower credibility value $=-.018$; Master: $\hat{\mu}_{\rho}=-.069,90 \%$ upper credibility value $=.088$ ). For investigating the moderator country of origin, we classified six different regions of origin: Africa, Australia, Europe, North America, East Asia, and Middle East. There was no study investigating the relationship between grades and Extraversion in Africa or the Middle East. The differences between the validities for Extraversion from the other regions were significant $(k=54, Q=50.115, d f=3$, $p<.00455)$ with substantial positive validity only for East Asian studies $\left(k=10, \hat{\mu}_{\rho}=.356\right)$. Table 6 shows results for the four regions in detail.

Similar results were found when calculating moderator analyses with the approach of Viechtbauer (described in this issue). Again, the country of the study was detected as moderator variable $(Q=36.32, d f=3, p<.001)$. The $95 \%$ confidence interval indicated a statistically significant association between Extraversion and grades only for East Asia $\left(\hat{\mu}_{\rho}=.383 ; C I=(.250, .502)\right)$. The results for the study period moderator (Bachelor versus Master, basic vs. main studies) are also given here, although this moderator just failed to be significant $(Q=8.00, d f=1, p=.0047)$. Nevertheless, the moderator can account for approximately $29 \%$ of the heterogeneity. The average correlation was positive for Bachelor and basic studies $\left(k=27, \hat{\mu}_{\rho_{\Lambda}}=.083\right)$ and negative for Master and main studies $\left(k=5, \mu_{\rho}=-.081\right)$, 
Table 7. Results of the meta-analysis for the Big Five personality traits and the criterion grades calculated by W. Viechtbauer (see method described in this issue)

\begin{tabular}{llllllllll}
\hline & $N$ & $k$ & \multicolumn{1}{l}{$M$} & $\hat{\mu}_{\rho}$ & $\hat{\sigma}_{\rho}$ & $95 \% C I_{R E}$ for $\mu_{\rho}$ & $95 \% C I_{R E}$ for $\sigma_{\rho}$ & $90 \% C V$ for $\rho_{\mathrm{i}}$ & $\mathrm{I}^{2}$ \\
\hline Neuroticism & 14653 & 59 & -.044 & -.038 & .123 & {$[-.080, .004]$} & {$[.094, .178]$} & .125 & $65 \%$ \\
Extraversion & 12424 & 54 & .011 & -.004 & .211 & {$[-.070, .062]$} & {$[.191, .313]$} & .275 & $82 \%$ \\
Openness to Experience & 14942 & 41 & .083 & .115 & .134 & {$[.064, .166]$} & {$[.106, .210]$} & -.065 & $76 \%$ \\
Agreeableness & 12452 & 34 & .041 & .060 & .095 & {$[.015, .104]$} & {$[.057, .146]$} & -.067 & $57 \%$ \\
Conscientiousness & 10855 & 41 & .216 & .279 & .124 & {$[.230, .326]$} & {$[.088, .168]$} & .108 & $73 \%$ \\
\hline
\end{tabular}

Notes: $N=$ overall sample size; $k=$ number of independent samples; $M=$ mean observed correlation; $\hat{\mu}_{\rho}=$ sample size weighted and corrected validity; $\hat{\sigma}_{\rho}=$ estimated SD of $\rho ; 95 \% C I_{R E}$ for $\mu_{\rho}=$ confidence interval for $\mu_{\rho}$ with $p=.95$, random effects model; $95 \% C I_{R E}$ for $\sigma_{\rho}$ confidence interval for $\sigma_{\rho}$ with $p=.95$, random effects model; $90 \% C V=$ credibility value: lower limit for positive $\mu_{\rho}$-values, upper limits for negative $\mu_{\rho}$-values of $90 \%$-credibility interval; $\mathrm{I}^{2}=$ total amount of variability in the correlation coefficients due to heterogeneity (in $\%$ )

although the difference between the two is not very large. Moreover, the $95 \%$ confidence interval for $\hat{\mu}_{\rho}$ was equal to $(.038, .128)$ for Bachelor and basic studies and equal to $(-.184, .023)$ for Master and main studies. Therefore, there is a hint of a slightly positive association for Bachelor and basic studies, but the average correlation is not significantly different from zero for Master and main studies. Given that this moderator is already not statistically significant, this finding is also not robust to imprecision in the estimated amount of residual heterogeneity. Table 7 shows metaanalytic results calculated by Wolfgang Viechtbauer.

\section{Discussion}

\section{Big Five and College Grades}

We expected substantial validity coefficients of Conscientiousness, Openness to Experience and Extraversion for college grades. Concerning the relationship between grades and Emotional Stability or grades and Agreeableness, we hypothesized small positive validities. Our hypotheses only fit for Conscientiousness. Though Agreeableness is correlated, in average, slightly positively with academic achievement, the effect size is very low and cannot be generalized. Conscientiousness shows the strongest validity for academic achievement as measured by college grades. The validity coefficient of .269 indicates that 7.2 percent of the criterion measure variance can be explained by this trait. The credibility interval indicates that the validity can be generalized although the percentage of variance accounted for by the artifacts is rather low. Conscientiousness is seen as a personality trait with an explicit behavioral meaning (De Raad \& Schouwenburg, 1996). It covers many facets that have high face validity for college success: the drive to accomplish something, being organized, efficient, systematic, orderly, and steady. In earlier studies on noncognitive factors in learning and education, facets of Conscientiousness have already been identified as crucial. Spearman (1927) postulated some 80 years ago that noncognitive factors like perseverative behavior, volitional behavior, and inertia determine intelligent behavior. The question of how Conscientiousness causes better grades in relation to general mental ability (GMA) has not yet been answered. Results provided by Mount, Barrick, and Strauss (1999), Schmidt and Hunter (1998), and Bobko, Roth, and Potosky (1999) support the hypothesis of a noninteractive effect of GMA and Conscientiousness in the work domain. According to this assumption, an incremental validity of Conscientiousness over and above GMA, as Schmidt and Hunter (1998) found for job performance, can be expected for academic performance as well (Conard, 2006; Ridgell \& Lounsbury, 2004).

As we investigated bivariate correlation coefficients, potential predictor redundancies were not taken into account. To estimate the individual amount of validity when two or more predictors are used at the same time, incremental validity has to be calculated. For this purpose, intercorrelations between predictors also have to be reported. Even though Conscientiousness is a valid predictor for academic achievement, hierarchical regression analyses indicate only marginal incremental validities over and above high school GPA in predicting university grades: To obtain an estimate of the incremental validity, we assumed Conscientiousness would be associated with school grades to the same extent as with university grades. That is, the intercorrelation between school grades and Conscientiousness is .269. The validity of German high school GPA for university grades is $\hat{\mu}_{\rho}=.525$ (Trapmann et al., 2007). Adjusted $R^{2}$ increased from .275 to .293 when adding Conscientiousness to high school GPA into the regression. That is, $1.8 \%$ of the variance in university grades is additionally explained when using Conscientiousness and high school GPA instead of high school GPA alone. Thus, the benefit of using Conscientiousness scores in addition to high school GPA for university admission is fairly small.

Contrary to our hypotheses, Extraversion and Openness to Experience do not predict college grades. Meta-analytic results showed no substantial validity of Extraversion for college grades $\left(\hat{\mu}_{\rho}=-.059\right)$ with a $90 \%$ credibility value beyond zero. However, moderator-analyses indicate significant cultural differences for this result. Even though sample sizes are rather small, we conclude that being extraverted facilitates academic success in some cultures (e.g., Eastern Asia) but rather interferes in others (Australia). The validity of Openness to Experience $\left(\hat{\mu}_{\rho}=.133\right)$ for college 
grades is not as low as the validity of Extraversion, but fails to be generalizable $(90 \%$ credibility value $<0$ and only $23.3 \%$ variance accounted for by artifacts). Moderator variables that could be checked for Openness to Experience failed to show significant influences in this relation. Openness to Experience as a construct may be too broad to predict academic success, and some facets are substantially valid while others are not. Meta-analyses of the lower-level traits openness to feelings, openness to actions, openness to ideas, and openness to values showed substantial validities with college grades only for the facet openness to ideas $\left(\hat{\mu}_{\rho}=.152, k=13\right.$; Trapmann, Hell, Hirn, Weigand \& Schuler, 2005). Only a small amount of variance is accounted for by artifacts (19.5\%), but credibility and confidence intervals do not include zero. Even though sample size is small, we regard these findings as a possible explanation for different validities of Openness to Experience for academic achievement. Griffin and Hesketh (2004) also reported differential validities of openness facets for the prediction of job performance.

In contrast to our hypothesis, Neuroticism does not seem to be an important predictor of academic grades. The weighted and corrected mean correlation ranges around zero $\left(\mu_{\rho}=-.003\right)$. Meta-analytic results suggest that the different facets of Neuroticism (anxiety, angry hostility, depression, self-consciousness, impulsiveness, and vulnerability) are correlated diversely with academic achievement. Trapmann et al. (2005) presented validities for all six facets of Neuroticism. Two facets (anxiety and angry hostility) show no validity for college grades $\left(k=13\right.$ and $k=5 ; \mu_{\rho}=$ .002 and $\left.\hat{\mu}_{\rho}=-.070\right)$. Contrariwise, results with respect to the other four facets (depression, self-consciousness, impulsiveness, and vulnerability) suggest small but significant validities with $\hat{\mu}_{\rho}$ ranging from -.12 to -.16 ( $k$ ranging from 4 to 19). None of the confidence intervals includes zero. However, given the small number of included coefficients further conclusions should be drawn carefully.

\section{Big Five and Retention}

Concerning the retention criterion we expected a low positive validity for Conscientiousness and a low negative validity for Openness to Experience. The first hypothesis could not be tested because we could not draw a sufficient number of studies focusing on this particular aspect. Only two coefficients for this relationship were obtained, with an unweighted uncorrected mean of $r=.140(S D=.028)$ and $\hat{\mu}_{\rho}=.177$ (corrected for sampling error as well as for error of measurement in both, predictor and criterion). The $95 \%$ confidence interval does not contain zero. This may be a hint in the direction of our hypothesis. The expected negative validity of Openness to Experience was not supported. The coefficient is almost zero $\left(\mu_{\rho}=.031\right)$. However, this result includes only four correlation coefficients and, therefore, may not be reliable. Moderator analyses could not be applied for this reason, either. Moreover, no infor- mation is available on whether the lower-level traits are correlated in different ways with college retention. Further research is needed concerning retention vs. attrition as a criterion of success. Validity coefficients with respect to Neuroticism and Extraversion were negligible. These results are similar to the results found in the field of job turnover and job tenure: In their meta-analysis, Barrick and Mount (1991) found no substantial validity coefficients for this criterion type.

\section{Big Five and Satisfaction}

We hypothesized that all five trait domains are valid predictors for satisfaction and that among those, Emotional Stability (Neuroticism) is the strongest predictor. These hypotheses were partly supported. Emotional Stability showed a strong relationship with satisfaction. Its validity for academic satisfaction is comparable to its validity for job and life satisfaction (DeNeve \& Cooper, 1998; Judge et al., 2002). Neuroticism was as strongly related with academic satisfaction $\left(\hat{\mu}_{\rho}=-.369\right)$ as with job satisfaction and life satisfaction (Judge et al., 2002). The relationship between satisfaction and Neuroticism is not only stronger than the associations between satisfaction and the other Big Five factors, but also stronger than the correlation between satisfaction and interest congruence (Tsabari, Tziner, \& Meir, 2004). One reason for this relationship might be that satisfaction or dissatisfaction is often an element of the definition of Neuroticism. Items mentioned to tap Neuroticism overlap with aspects of the satisfaction domain (e.g., IPIP: "I am very pleased with myself," "I dislike myself," Goldberg et al., 2006; NEO-PI-R: "Sometimes I feel completely worthless," "I have a low opinion of myself," Costa \& McCrae, 1992b). Additionally, the validity coefficient obtained might be partly due to the result of common method variance because predictor and criterion are measured by questionnaire (Schuler, 2000). Thus, the high correlation coefficient between Neuroticism and the satisfaction criterion might be due to the validity of the predictor, overlapping constructs, or common method variance.

\section{Conclusions and Implications}

In summary, the validity of Conscientiousness for academic grades at the university level has been shown to be reliable and is comparable with the validity for job performance (Barrick et al., 2001). Conscientiousness emerged as the only Big Five trait that shows a substantial validity for college or university grades. As we did not find significant moderator effects for the validity of Conscientiousness, we conclude that Conscientiousness is an important trait for academic achievement without substantial differences for study majors, culture, age or other investigated moderator variables. Conscientiousness might be useful for student admission if it was measured without distorting bi- 
ases (e.g., referring to socially desirable responding). Typically, personality traits are measured by self-reports. The disadvantage of using self-reports is that they are amenable to impression management, that is, coachable and fakable. Goldberg (2001) suggested that this is a problem especially in university admission, which has very high stakes for applicants. Therefore, in a college admission context, it is likely that validity is reduced because of faking. Results obtained in studies with job performance as criterion (Ones \& Viswesvaran, 1998), showing that validity is not reduced by faking, cannot be easily transferred, because items may become familiar and applicants may - more often than in occupational settings - be professionally coached.

There are methods designed to minimize faking (peer ratings, skills or performance "objective" measures, "lie scales," warnings from the proctor, subtle-items method, multidimensional forced-choice method) but they are not evaluated sufficiently to be applied to university admission (Kyllonen et al., 2005). Furthermore, "The coaching industry would quickly crack the code on any noncognitive measure and translate that into a successful strategy for defeating the measure if it were a self-assessment" (Kyllonen et al., 2005, p. 177). This rather pessimistic view is strengthened by recent studies concerning the fakability of situational judgment tests, as well (Peeters \& Lievens, 2005). Apparently, it is a current effort to develop so-called "objective" performance measures of personality traits (e.g., achievement motivation: Schmidt-Atzert, 2004; James, 1998; resilience: Ortner, Kubinger, Schrott, Radinger, \& Litzenberger, 2006). These measures promise not to be fakable and coachable. Unfortunately, they have not been tested yet on large scales and the validities of these procedures still remain unclear. Moreover, we do not know whether such tests can be developed for all traits of interest in the context of university admission, whether they would be valid for one or more success criteria, or whether they would be coachable in case they become familiar. It is not possible to answer these questions with our results. As mentioned earlier, none of the included studies presented personality measures in real selection conditions.

To avoid problems aligned to the method of personality inventories it might be promising to apply other methods, such as structured interviews, to assess personal information of the applicants. In a recent meta-analysis, structured admission interviews showed a generalizable validity of .21 (Hell, Trapmann, Weigand, \& Schuler, 2007). It is conceivable to operationalize auspicious personality domains like Conscientiousness and hierarchic lower facets in admission interviews to use this measure as a complement to other admission criteria such as school grades and achievement tests.

Another potential application of using personality measures might be student guidance and development. Students who score low on Conscientiousness could benefit from supporting interventions. Although personality traits are rather stable over time, there is evidence that they evolve and change in young adults (Roberts \& DelVecchio,
2000; McCrae \& Costa, 1994). Kyllonen et al. (2005) suggested that it might be useful to help applicants to choose the right educational program by comparing an applicant's personality profile to the profiles of successful graduates. The usefulness of this kind of guidance has not yet been proven. In the light of our results, we would not expect large differences in personality profiles between successful graduates of different kinds of universities or majors. Rather, we would expect successful graduates scoring generally higher on Conscientiousness scales.

Maybe personality traits will become more relevant to academic success in the future when we think of e-learning situations. If e-learning was the ordinary way to study in the future, students would get less personal support and individual differences would become more and more important. On the other hand it is not very likely that personality variables will ever exceed the validity of achievement tests and school grades for college grades. These predictors explain a large portion of the variance of this criterion. It seems promising for further studies to broaden the criterion domain and reconsider organizational citizenship behavior (Organ, 1988), interpersonal study aspects, and variables like personal growth in their investigation.

\section{Acknowledgments}

The paper was written as part of the project "Student Selection" ("Studierendenauswahl") commissioned by the Landesstiftung Baden-Württemberg and the Stifterverband für die deutsche Wissenschaft.

We would like to thank Nambury Raju†, Stephan J. Rustenbach, Frank L. Schmidt, and Ralf Schulze for thoughtful comments on some meta-analytic questions of detail and Wolfgang Viechtbauer for calculating moderator analyses with his new method.

\section{References}

References marked with an asterisk $(*)$ indicate studies included in the meta-analysis (see Table 1).

*Barchard, K.A. (2003). Does emotional intelligence assist in the prediction of academic success? Educational and Psychological Measurement, 63, 840-858.

*Bauer, K.W., \& Liang, Q. (2003). The effect of personality and precollege characteristics on first-year activities and academic performance. Journal of College Student Development, 44, 277-290.

Barrick, M.R., \& Mount, M.K. (1991). The Big Five personality dimensions and job performance: A meta-analysis. Personnel Psychology, 44, 1-26.

Barrick, M.R., Mount, M.K., \& Judge, T.A. (2001). Personality and performance at the beginning of the new millennium: What do we know and where do we go next? International Journal of Selection and Assessment, 9, 9-30. 
Bobko, P., Roth, P.L., \& Potosky, D. (1999). Derivation and implications of a meta-analytic matrix incorporating cognitive ability, alternative predictors, and job performance. Personnel Psychology, 52, 561-589.

*Brandstätter, H., \& Farthofer, A. (2002). Studienerfolgsprognose - konfigurativ oder linear additiv? [Prediction of academic success - Configurative or linearly additive?]. Zeitschrift für Differentielle und Diagnostische Psychologie, 23, 381-391.

Burton, N.W., \& Ramist, L. (2001). Predicting success in college: SAT studies of classes graduating since 1980. The College Board Research Report, 2001-2002. New York: College entrance Examination Board.

*Busato, V.V., Prins, F.J., Elshout, J.J., \& Hamaker, C. (2000). Intellectual ability, learning style, personality, achievement motivation, and academic success of psychology students in higher education. Personality and Individual Differences, 29, 1057-1068.

Camara, W.J. (2005). Broadening criteria of college success and the impact of cognitive predictors. In W.J. Camara \& E.W. Kimmel (Eds.), Choosing students: Higher education admissions tools for the 21st century (pp. 53-79). Mahwah, NJ: Erlbaum.

Cattell, R.B., Eber, H.W., \& Tatsuoka, M.M. (1970). Handbook for the 16 Personality Factor Questionnaire (16PF). Champaign, IL: Institute for Personality and Ability Testing.

*Chamorro-Premuzic, T., \& Furnham, A. (2003a). Personality predicts academic performance: Evidence from two longitudinal university samples. Journal of Research in Personality, 37, 319-338.

*Chamorro-Premuzic, T. \& Furnham, A. (2003b). Personality traits and academic examination performance. European Journal of Personality, 17, 237-250.

*Colquitt, J.A., \& Simmering, M.J. (1998). Conscientiousness, goal orientation, and motivation to learn during the learning process: A longitudinal study. Journal of Applied Psychology, 83, 654-665.

Conard, M.A. (2006). Aptitude is not enough: How personality and behavior predict academic performance. Journal of Research in Personality, 40, 339-346.

Costa, P.T., \& McCrae, R.R. (1991). The NEO Five Factor Inventory. Odessa, FL: Psychological Assessment Resources.

Costa, P.T., \& McCrae, R.R. (1992a). Four ways five factors are basic. Personality and Individual Differences, 13, 653-665.

Costa, P.T., \& McCrae, R.R. (1992b). Revised NEO Personality Inventory and NEO Five Factor Inventory Professional manual. Odessa; FL: Psychological Assessment Resources.

*De Fruyt, F., \& Mervielde, I. (1996). Personality and interests as predictors of educational streaming and achievement. European Journal of Personality, 10, 405-425.

DeNeve, K.M., \& Cooper, H. (1998). The happy personality: A meta-analysis of 137 personality traits and subjective well-being. Psychological Bulletin, 124, 197-229.

De Raad, B., \& Schouwenburg, H.C. (1996). Personality in learning and education: A review. European Journal of Personality, 10, 303-336.

Digman, J.M. (1990). Personality structure: Emergence of the five-factor model. Annual Review of Psychology, 41, 417-440.

*Diseth, A. (2003). Personality and approaches to learning as predictors of academic achievement. European Journal of Personality, 17, 143-155.

*Dollinger, S.J., \& Orf, L.A. (1991). Personality and performance in "personality": Conscientiousness and openness. Journal of Research in Personality, 25, 276-284.

Dudley, N.M., Orvis, K.A., Lebiecki, J.E., \& Cortina, J.M. (2006). A meta-analytic investigation of Conscientiousness in the prediction of job performance: Examining the intercorrelations and the incremental validity of narrow traits. Journal of Applied Psychology, 91, 40-57.

*Duff, A., Boyle, E., Dunleavy, K., \& Ferguson, J. (2004). The relationship between personality, approach to learning, and academic performance. Personality and Individual Differences, 36, 1907-1920.

*Dyer, E.D. (1987). Can university success and first-year job performance be predicted from academic achievement, vocational interest, personality, and biographical measures? Psychological Reports, 61, 655-671.

*Edwards, W.R., \& Schleicher, D.J. (2004). On selecting psychology graduate students: Validity evidence for a test of tacit knowledge. Journal of Educational Psychology, 96, 592-602.

Enright, M.K., \& Gitomer, D. (1989). Toward a description of successful graduate students. Princeton, NJ: Educational Testing Service.

Eysenck, H.J., \& Eysenck, S.B.G. (1968). The manual to the Eysenck Personality Inventory. San Diego: California.

Eysenck, H.J., \& Eysenck, S.B.G. (1975). The Eysenck Personality Questionnaire manual. London: Hodder \& Stoughton.

*Farsides, T., \& Woodfield, R. (2003). Individual differences and undergraduate academic success: The roles of personality, intelligence, and application. Personality and Individual Differences, 34, 1225-1243.

*Ferguson, E., James, D., O’Hehir, F., \& Sanders, A. (2003). Pilot study of the roles of personality, references, and personal statements in relation to performance over the 5 years of a medical degree. British Medical Journal, 326, 429-431.

Fleenor, J.W., \& Eastman, K. (1997). The relationship between the five-factor model of personality and the California Psychological Inventory. Educational and Psychological Measurement, 57, 698-703.

*Furnham, A., \& Chamorro-Premuzic, T. (2004). Personality and intelligence as predictors of statistics examination grades. Personality and Individual Differences, 37, 943-955.

*Furnham, A., Chamorro-Premuzic, T., \& McDougall, F. (2002). Personality, cognitive ability, and beliefs about intelligence as predictors of academic performance. Learning and Individual Differences, 14, 47-64.

*Furnham, A., \& Mitchell, J. (1991). Personality, needs, social skills, and academic achievement: A longitudinal study. Personality and Individual Differences, 12, 1067-1073.

*Gakhar, S.C. (1986). Intellectual and personality correlates of academic achievement of college students undertaking different courses. Indian Psychological Review, 30, 9-16.

*Giesen, H., Gold, A., Hummer, A., \& Jansen, R. (1986). Prognose des Studienerfolgs [Prediction of academic success]. Frankfurt am Main: Institut für Pädagogische Psychologie.

*Goff, M., \& Ackerman, P.L. (1992). Personality-intelligence relations: Assessment of typical intellectual engagement. Journal of Educational Psychology, 84, 537-552.

Goldberg, L.R. (2001, June). Frozen by success: Why we don't know nearly enough about the relations between personality attributes and academic performance. Remarks delivered at the E.T.S. Workshop: Applications to new constructs, Educational Testing Service, Princeton, NJ. 
Goldberg, L.R., Johnson, J.A., Eber, H.W., Hogan, R., Ashton, M.C., Cloninger, C.R. et al. (2006). The International Personality Item Pool and the future of public-domain personality measures. Journal of Research in Personality, 40, 84-96.

Gough, H.G. (1975). California Psychological Inventory (CPI): Manual. Palo Alto, CA: Consulting Psychologists Press, Inc.

*Gough, H.G., \& Lanning, K. (1986). Predicting grades in college from the California Psychological Inventory. Educational and Psychological Measurement, 46, 205-213.

*Gray, E.K., \& Watson, D. (2002). General and specific traits of personality and their relation to sleep and academic performance. Journal of Personality, 70, 177-206.

Griffin, B., \& Hesketh, B. (2004). Why Openness to Experience is not a good predictor of job performance. International Journal of Selection and Assessment, 12, 243-251.

Hell, B., Trapmann, S., \& Schuler, H. (2007). Eine Metaanalyse der Validität von fachspezifischen Studierfähigkeitstests im deutschsprachigen Raum [A meta-analytic investigation of subject-specific admission tests in German-speaking countries]. Empirische Pädagogik, 21, 251-270.

Hell, B., Trapmann, S., Weigand, S., \& Schuler, H. (2007). Die Validität von Auswahlgesprächen im Rahmen der Hochschulzulassung - eine Metaanalyse [The validity of admission interviews - A meta-analysis]. Psychologische Rundschau, 58, 93-102.

Hogan, R. (1986). Hogan Personality Inventory. Minneapolis, MN: National Computer Systems.

*Hörschgen, H., Cierpka, R., Friese, M., \& Steinbach, R. (1993). Erfolg im Studium und Beruf [Academic and occupational success]. Universität Hohenheim - Institut für Betriebswirtschaftslehre.

*Hough, L.M. (1992). The Big Five personality variables - Construct confusions: Description versus prediction. Human Performance, 5, 139-155.

Hough, L.M., \& Ones, D.S. (2002). The structure, measurement, validity, and use of personality variables in industrial, work, and organizational psychology. In N. Anderson, D.S. Ones, H.K. Sinangil, \& C. Viswesvaran (Eds), Handbook of industrial, work, and organizational psychology, Volume 1: Personnel psychology (pp. 233-277). Thousand Oaks, CA: Sage Ltd.

Hunter, J.E., \& Schmidt, F.L. (2004). Methods of meta-analysis Correcting error and bias in research findings ( 2 nd ed.). Thousand Oaks, CA: Sage.

Hunter, J.E., Schmidt, F.L., \& Jackson, G.B. (1982). Meta-analysis: Cumulating research findings across studies. London: Sage.

*Huq, M., Rabman, M.M., \& Mahmud, S.H. (1986). Role of neuroticism, psychoticism, and extraversion in academic achievement. Asian Journal of Psychology and Education, 17, 1-6.

James, L.R. (1998). Measurement of personality via conditional reasoning. Organizational Research Methods, 1, 131-163.

Judge, T.A., Heller, D., \& Mount, M.K. (2002). Five-factor model of personality and job satisfaction: A meta-analysis. Journal of Applied Psychology, 87, 530-541.

*Kahn, J.H., Nauta, M.M., Gailbreath, R.D., Tipps, J., \& Chartrand, J.M. (2002). The utility of career and personality assessment in predicting academic progress. Journal of Career Assessment, 10, 3-23.

*Kalsbeek, D. (2003). Campus retention: The MBTI in institutional self-studies. In J. Provost \& S. Anchors (Eds.), Using the MBIT instrument in colleges and universities (pp. 87-122). Gainesville: Center for Applications of Psychological Type.

*King, A.R., \& Bailly, M.D. (2002). MMPI-2 predictors of academic performance. Journal of Psychoeducational Assessment, 20, 258-267.

Kuncel, N.R., Hezlett, S.A., \& Ones, D.S. (2001). A comprehensive meta-analysis of the predictive validity of the graduate record examinations: Implications for graduate student selection and performance. Psychological Bulletin, 127, 162-181.

Kuncel, N.R., Hezlett, S.A., \& Ones, D.S. (2004). Academic performance, career potential, creativity, and job performance: Can one construct predict them all? Journal of Personality and Social Psychology, 86, 148-161.

Kyllonen, P.C., Walters, A.M., \& Kaufman, J.C. (2005). Noncognitive constructs and their assessment in graduate education: A review. Educational Assessment, 10, 153-184.

*Lipton, A., Huxham, J., \& Hamilton, D. (1984). Predictors of success in a cohort of medical students. Medical Education, $18,203-210$.

*Lounsbury, J.W., Sundstrom, E., Loveland, J.M., \& Gibson, L.W. (2003). Intelligence, Big Five personality traits, and work drive as predictors of course grade. Personality and Individual Differences, 35, 1231-1239.

*Lufi, D., Parish-Plass, J., \& Cohen, A. (2003). Persistence in higher education and its relationship to other personality variables. College Student Journal, 37, 50-59.

*McCabe, R.E., Blankstein, K.R., \& Mills, J.S. (1999). Interpersonal sensitivity and social problem-solving: Relations with academic and social self-esteem, depressive symptoms, and academic performance. Cognitive Therapy and Research, 23, 587-604.

McCrae, R.R., \& Costa, P.T. Jr. (1994). The stability of personality: Observations and evaluations. Current Directions in Psychological Science, 3, 173-175.

*McIlroy, D., \& Bunting, B. (2002). Personality, behavior, and academic achievement: Principles for educators to inculcate and students to model. Contemporary Educational Psychology, 27, 326-337.

*McKenzie, J. (1989). Neuroticism and academic achievement: The Furneaux Factor. Personality and Individual Differences, 10, 509-515.

*McKenzie, J., Taghavi-Khonsary, M., \& Tindell, G. (2000). Neuroticism and academic achievement: The Furneaux Factor as a measure of academic rigor. Personality and Individual Differences, 29, 3-11.

*McKenzie, J., \& Tindell, G. (1993). Anxiety and academic achievement: Further Furneaux Factor findings. Personality and Individual Differences, 15, 609-617.

*McKenzie, K., \& Gow, K. (2004). Exploring the first year academic achievement of school leavers and mature-age students through structural equation modeling. Learning and Individual Differences, 14, 107-123.

*Mehta, P., \& Kumar, D. (1985). Relationships of academic achievement with intelligence, personality, adjustment, study habits, and academic motivation. Journal of Personality and Clinical Studies, 1, 57-68.

*Mendoza, J.L., \& Reinhardt, R.N. (1991). Validity generalization procedures using sample-based estimates: A comparison of six procedures. Psychological Bulletin, 110, 596-610.

*Mershon, B., \& Gorsuch, R.L. (1988). Number of factors in the personality sphere: Does increase in factors increase predict- 
ability of real-life criteria? Journal of Personality and Social Psychology, 55, 675-680.

*Moon, S.M., \& Illingworth, A.J. (2005). Exploring the dynamic nature of procrastination: A latent growth curve analysis of academic procrastination. Personality and Individual Differences, 38, 297-309.

*Morris, L.W., \& Carden, R.L. (1981). Relationship between locus of control and extraversion-introversion in predicting academic behavior. Psychological Reports, 48, 799-806.

Mount, M.K., Barrick, M.R., \& Strauss, J.P. (1999). The joint relationship of conscientiousness and ability with performance: Test of the interaction hypothesis. Journal of Management, 25, 707-721.

*Murtha, T.C., Kanfer, R., \& Ackerman, P.L. (1996). Toward an interactionist taxonomy of personality and situations: An integrative situational- dispositional representation of personality traits. Journal of Personality and Social Psychology, 71, 193-207.

*Musgrave-Marquart, D., Bromley, S.P., \& Dalley, M.B. (1997). Personality, academic attribution, and substance use as predictors of academic achievement in college students. Journal of Social Behavior and Personality, 12, 501-511.

*Nath, L.M. (1987). Role of personality and interest for students' success in medical course. Psychological Research Journal, $11,54-63$.

Novy, D.M., Kopel, K.F., \& Swank, P.R. (1996). Psychometrics of oral examinations for psychology licensure: The Texas examination as an example. Professional Psychology: Research and Practice, 27, 415-417.

*Okun, M.A., \& Finch, J.F. (1998). The Big Five personality dimensions and the process of institutional departure. Contemporary Educational Psychology, 23, 233-256.

Ones, D.S., \& Viswesvaran, C. (1998). The effects of social desirability and faking on personality and integrity assessment for personnel selection. Human Performance, 11, 245-269.

*Oosthuizen, S. (1990). Graphology as predictor of academic achievement. Perceptual and Motor Skills, 71, 715-721.

Organ, D.W. (1988). Organizational citizenship behavior: The good soldier syndrome. Lexington, MA: D.C. Heath and Company.

Ortner, T.M., Kubinger, K.D., Schrott, A., Radinger, R., \& Litzenberger, M. (2006). Belastbarkeits-Assessment: computerisierte Objektive Persönlichkeits-Testbatterie - Deutsch (BAcO-D) [Assessment of Resilience: Computer-based objective personality test - German]. Test: Software und Manual. Frankfurt/M.: Harcourt Test Services.

Ostendorf, F., \& Angleitner, A. (2004). NEO-Persönlichkeitsinventar nach Costa \& McCrae [NEO Personality Inventory according to Costa \& McCrae]. Göttingen: Hogrefe.

*Oswald, F.L., Schmitt, N., Kim, B.H., Ramsay, L.J., \& Gillespie, M.A. (2004). Developing a biodata measure and situational judgment inventory as predictors of college student performance. Journal of Applied Psychology, 89, 187-207.

*Paunonen, S.V., \& Ashton, M.C. (2001). Big Five predictors of academic achievement. Journal of Research in Personality, 35, 78-90.

*Peeters, H., \& Lievens, F. (2005). Situational judgment tests and their predictiveness of college students' success: The influence of faking. Educational and Psychological Measurement, 65, 70-89.

*Peng, R., Khaw, H.H., \& Edariah, A.B. (1995). Personality and performance of preclinical medical students. Medical Education, 29, 283-288.

Potosky, D., Bobko, P., \& Roth, P.L. (2005). Forming composites of cognitive ability and alternative measures to predict job performance and reduce adverse impact: Corrected estimates and realistic expectations. International Journal of Selection and Assessment, 13, 304-315.

Raju, N.S., \& Fleer, P.F. (2003). VG2M: A computer program for conducting validity generalization analysis. Chicago, IL: Illinois Institute of Technology.

Raju, N.S., Burke, M.J., Normand, J., \& Langlois, G.M. (1991). A new meta-analytic approach. Journal of Applied Psychology, 76, 432-446.

Reilly, R.R. (1976). Factors in graduate student performance. American Educational Research Journal, 13, 125-138.

*Ridgell, S.D., \& Lounsbury, J.W. (2004). Predicting academic success: General intelligence, Big Five personality traits, and work drive. College Student Journal, 38, 607-619.

Robbins, S.B., Lauver, K., Le, H., Davis, D., Langley, R., \& Carlstrom, A. (2004). Do psychosocial and study skill factors predict college outcomes? A meta-analysis. Psychological Bulletin, 130, 261-288.

Roberts, B.W., \& DelVecchio, W.F. (2000). The rank-order consistency of personality traits from childhood to old age: A quantitative review of longitudinal studies. Psychological Bulletin, 126, 3-25.

Rossier, J., Meyer de Stadelhofen, F., \& Berthoud, S. (2004). The hierarchical structures of the NEO-PI-R and the 16PF5. European Journal of Psychological Assessment, 20, 27-38.

*Rothstein, M.G., Paunonen, S.V., Rush, J.C., \& King, G.A. (1994). Personality and cognitive ability predictors of performance in graduate business school. Journal of Educational Psychology, 86, 516-530.

Salgado, J.F. (1997). The five, factor model of personality and job performance in the European Community. Journal of Applied Psychology, 82, 30-43.

*Schmidt-Atzert, L. (2005). Prädiktion von Studienerfolg bei Psychologiestudenten [Prediction of academic success in psychology students]. Psychologische Rundschau, 56, 131-133.

Schmidt-Atzert, L. (2004). Objektiver Leistungsmotivations-Test OLMT [Objective Achievement-Motivation-Test OLMT]. Mödling: Schuhfried.

Schmidt, F.L., \& Hunter, J.E. (1998). The validity and utility of selection methods in personnel psychology: Practical and theoretical implications of 85 years of research findings. Psychological Bulletin, 124, 262-274.

Schmidt, F.L., \& Le, H. (2004). Software for the Hunter-Schmidt meta-analysis methods. University of Iowa, Department of Management and Organization, Iowa City, IA.

Schuler, H. (2000). Das Rätsel der Merkmals-Methoden-Effekte: Was ist "Potential" und wie läßt es sich messen? [The riddle of trait-method-effects: What is "potential" and how to measure it?] In L. von Rosenstiel \& T. Lang-von Wins (Eds.), Perspektiven der Potentialbeurteilung [Perspectives on the assessment of potentials] (pp. 27-71). Göttingen: Hogrefe.

Schuler, H., Funke, U., \& Baron-Boldt, J. (1990). Predictive validity of school grades: A meta-analysis. Applied Psychology: An International Review, 39, 89-103.

Schulze, R. (2004). Meta-analysis: A comparison of approaches. Cambridge, MA: Hogrefe \& Huber.

*Schurr, K.T., Ruble, V.E., Palomba, C., Pickerill, B., \& Moore, 
D. (1997). Relationships between the MBTI and selected aspects of Tinto's model for college attrition. Journal of Psychological Type, 40, 31-42.

Seipp, B. (1991). Anxiety and academic performance: A metaanalysis of findings. Anxiety Research, 4, 27-41.

Spearman, C. (1927). Abilities of man. London: Macmillan.

Steers, R.M., \& Braunstein, D.L. (1976). A behaviorally based measure of manifest needs in work settings. Journal of Vocational Behavior, 14, 251-266.

Taber, T.D., \& Hackman, J.D. (1976). Dimensions of undergraduate college performance. Journal of Applied Psychology, 61, 546-558.

Tett, R.P., Jackson, D.N., \& Rothstein, M. (1991). Personality measures as predictors of job performance: A meta-analytic review . Personnel Psychology, 44, 703-742.

Trapmann, S. (2007). Mehrdimensionale Studienerfolgsprognose: Die Bedeutung kognitiver, temperamentsbedingter und motivationaler Prädiktoren für verschiedene Erfolgskriterien des Studienerfolgs [Multidimensional prediction of academic achievement: The impact of cognitive and noncognitive predictors for different kinds of success criteria]. Dissertation in preparation, University of Hohenheim.

Trapmann, S., Hell, B., Hirn, J.-O.W., Weigand, S., \& Schuler, H. (2005, September). Psychologische Konstrukte als Prädiktoren des Studienerfolgs - eine Metaanalyse [Psychological constructs as predictors of academic success - A meta-analysis]. Vortrag auf der 8. Arbeitstagung der Fachgruppe Differentielle Psychologie, Persönlichkeitspsychologie und Psychologische Diagnostik der Deutschen Gesellschaft für Psychologie in Marburg. [Paper presented at the 8th Conference of the Department of Psychology of Individual Differences and Psychological Assessment of the German Society of Psychology in Marburg].

Trapmann, S., Hell, B., Weigand, S., \& Schuler, H. (2007). Die Validität von Schulnoten zur Vorhersage des Studienerfolgs eine Metaanalyse [The validity of high-school grades for academic achievement - A meta-analysis]. Zeitschrift für Pädagogische Psychologie, 21, 11-27.

*Tross, S.A., Harper, J.P., Osher, L.W., \& Kneidinger, L.M (2000). Not just the usual cast of characters: Using personality to predict college performance and retention. Journal of College Student Development, 41, 323-334.
Tsabari, O., Tziner, A., \& Meir, E.I. (2004). Updated meta-analysis on the relationship between congruence and satisfaction. Journal of Career Assessment, 13, 216-232.

*Tutton, P.J. (1996). Psychometric test results associated with high achievement in basic science components of a medical curriculum. Academic Medicine, 71, 181-186.

*Upmanyu, V.V., Upmanyu, S., \& Vasudeva, P.N. (1980). Factor analytical study of measures of anxiety, neuroticism, psychoticism, intelligence, and academic achievement. Psychological Studies, 25, 90-97.

Viswesvaran, C., \& Ones, D.S. (2000). Measurement error in Big Five factors personality assessment: Reliability generalization across studies and measures. Educational and Psychological Measurement, 60, 224-235.

*Watkins, D., \& Astilla, E. (1980). Intellective and nonintellective predictors of academic achievement at a Filipino university. Educational and Psychological Measurement, 40, 245-249.

Wass, V., Wakeford, R., Neighbor, R., \& Van der Vleuten, C. (2003). Achieving acceptable reliability in oral examinations: An analysis of the Royal College of General Practitioners membership examination's oral component. Medical Education, 37, 126-131.

*Watson, D., \& Clark, L.A. (1993). Behavioral disinhibition versus constraint: A dispositional perspective. In D.M. Wegner \& J.W. Pennebaker (Eds.), Handbook of mental control (pp. 506-527). Englewood Cliffs, NJ: Prentice-Hall, Inc.

Willingham, W.W. (1985). Success in college: The role of personal qualities and academic ability. New York: College Entrance Examination Board.

*Wolfe, R.N., \& Johnson, S.D. (1995). Personality as a predictor of college performance. Educational \& Psychological Measurement, 55, 177-185.

Sabrina Trapmann

University of Hohenheim

Department of Psychology (540F)

D-70593 Stuttgart

Germany

E-mail sabrina_trapmann@gmx.de 